\title{
Patterns in evolutionary origins of heme, chlorophyll a and isopentenyl diphosphate biosynthetic pathways suggest non- photosynthetic periods prior to plastid replacements in dinoflagellates
}

\author{
Eriko Matsuo $^{1}{ }^{,}$Yuji Inagaki ${ }^{\text {Corresp. } 1,2}$ \\ ${ }^{1}$ Graduate School of Biological and Environmental Sciences, University of Tsukuba, Tsukuba, Ibaraki, Japan \\ 2 Center for Computational Sciences, University of Tsukuba, Tsukuba, Ibaraki, Japan \\ Corresponding Author: Yuji Inagaki \\ Email address: yuji@ccs.tsukuba.ac.jp
}

Background: The ancestral dinoflagellate most likely established a peridinin-containing plastid, which have been inherited to the extant photosynthetic descendants. However, kareniacean dinoflagellates and Lepidodinium species were known to bear "non-canonical" plastids lacking peridinin, which were established through haptophyte and a green algal endosymbioses, respectively. For plastid function and maintenance, the aforementioned dinoflagellates were known to use nucleus-encoded proteins vertically inherited from the ancestral dinoflagellates (vertically inherited- or VI-type), and those acquired from non-dinoflagellate organisms (including the endosymbiont). These observations indicated that the proteomes of the non-canonical plastids derived from a haptophyte and a green alga were modified by "exogenous" genes acquired from non-dinoflagellate organisms. However, there was no systematic evaluation addressing how "exogenous" genes reshaped individual metabolic pathways localized in a non-canonical plastid.

Results: In this study, we surveyed transcriptomic data from two kareniacean species (Karenia brevis and Karlodinium veneficum) and Lepidodinium chlorophorum, and identified proteins involved in three plastid metabolic pathways synthesizing chlorophyll a (Chl a), heme and isoprene. The origins of the individual proteins of our interest were investigated, and assessed how the three pathways were modified before and after the algal endosymbioses, which gave rise to the current non-canonical plastids. We observed a clear difference in the contribution of VI-type proteins across the three pathways. In both Karenia/Karlodinium and Lepidodinium, we observed a substantial contribution of VI-type proteins to the isoprene and heme biosynthesises. In sharp contrast, VI-type protein was barely detected in the Chl a biosynthesis in the three dinoflagellates.

Discussion: Pioneering works hypothesized that the ancestral kareniacean species had lost the photosynthetic activity prior to haptophyte endosymbiosis. The absence of VI-type proteins in the Chl a biosynthetic pathway in Karenia or Karlodinium is in good agreement with the putative nonphotosynthetic nature proposed for their ancestor. The dominance of proteins with haptophyte origin in the Karenia/Karlodinium pathway suggests that their ancestor rebuilt the particular pathway by genes acquired from the endosymbiont. Likewise, we here propose that the ancestral Lepidodinium likely experienced a non-photosynthetic period and discarded the entire $\mathrm{Chl}$ a biosynthetic pathway prior to the green algal endosymbiosis. Nevertheless, Lepidodinium rebuilt the pathway by genes transferred from phylogenetically diverse organisms, rather than the green algal endosymbiont. We explore the reasons why green algal genes were barely utilized to reconstruct the Lepidodinium pathway. 
1 Patterns in evolutionary origins of heme, chlorophyll $a$ and isopentenyl diphosphate

2 biosynthetic pathways suggest non-photosynthetic periods prior to plastid replacements in

3 dinoflagellates.

4

5 Eriko Matsuo $^{1}$, Yuji Inagaki ${ }^{1,2}$.

6

$7{ }^{1}$ Graduate School of Life and Environmental Sciences, University of Tsukuba, Tsukuba, Ibaraki,

8 Japan. ${ }^{2}$ Center for Computational Sciences, University of Tsukuba, Tsukuba, Ibaraki.

9

10 To whom correspondence should be addressed: Yuji Inagaki, yuji@ccs.tsukuba.ac.jp

11

12 


\section{Abstract}

Background: The ancestral dinoflagellate most likely established a peridinin-containing plastid, which has been inherited to the extant photosynthetic descendants. However, kareniacean dinoflagellates and Lepidodinium species were known to bear "non-canonical" plastids lacking peridinin, which were established through a haptophyte and a green algal endosymbioses, respectively. For plastid function and maintenance, the aforementioned dinoflagellates were known to use nucleus-encoded proteins vertically inherited from the ancestral dinoflagellates (vertically inherited- or VI-type), and those acquired from non-dinoflagellate organisms including the endosymbionts. These observations indicated that the proteomes of the non-canonical plastids derived from a haptophyte and a green alga were modified by "exogenous" genes acquired from non-dinoflagellate organisms. However, there was no systematic evaluation addressing how "exogenous" genes reshaped individual metabolic pathways localized in a non-canonical plastid.

Results: In this study, we surveyed transcriptomic data from two kareniacean species [Karenia (Ke.) brevis and Karlodinium (Kl.) veneficum] and Lepidodinium chlorophorum, and identified proteins involved in three plastid metabolic pathways synthesizing chlorophyll $a(\mathrm{Chl} a)$, heme and isopentenyl diphosphate (IPP). The origins of the individual proteins of our interest were investigated, and assessed how the three pathways were modified before and after the algal endosymbioses, which gave rise to the current non-canonical plastids. We observed a clear difference in the contribution of VI-type proteins across the three pathways. In both Ke. brevis/Kl. veneficum and L. chlorophorum, we observed a substantial contribution of VI-type proteins to the IPP and heme biosyntheses. In sharp contrast, VI-type protein was barely detected in the Chl $a$ biosynthesis in the three dinoflagellates. 
Discussion: Pioneering works hypothesized that the ancestral kareniacean species had lost the photosynthetic activity prior to haptophyte endosymbiosis. The absence of VI-type proteins in the Chl a biosynthetic pathway in Ke. brevis or Kl. veneficum is in good agreement with the putative non-photosynthetic nature proposed for their ancestor. The dominance of proteins with haptophyte origin in the Ke. brevis/Kl. veneficum pathway suggests that their ancestor rebuilt the particular pathway by genes acquired from the endosymbiont. Likewise, we here propose that the ancestral Lepidodinium likely experienced a non-photosynthetic period and discarded the Chl $a$ biosynthetic pathway mostly prior to the green algal endosymbiosis. In the subsequent evolution, $L$. chlorophorum rebuilt the pathway by genes transferred from phylogenetically diverse organisms, rather than the green algal endosymbiont. We explore the reasons why green algal genes were barely utilized to reconstruct the L. chlorophorum pathway.

\section{Introduction}

Dinoflagellates are aquatic unicellular eukaryotes belonging to one of major taxonomic groups of eukaryotes, Alveolata. About half species of dinoflagellates described to date are photosynthetic (Taylor et al, 2008). The vast majority of photosynthetic dinoflagellates harbor plastids containing chlorophylls $a$ and $c(\mathrm{Chl} a+c)$, which are remnants of a red algal endosymbiont captured by the common ancestor of dinoflagellates (Hoek et al, 1995; Janouškovec et al, 2010). Comparing to red alga-derived, Chl $a+c$-containing plastids in other eukaryotic algae, namely cryptophytes, chromerids (e.g., Chromera velia and Vitrella brassicaformis), stramenopiles, and haptophytes, the plastids in the vast majority of dinoflagellates are unique in containing a carotenoid called peridinin (Jeffrey et al, 1975; Zapata et al, 2012). However, some species are known to bear "non- 
61 canonical" plastids, which are distinctive from "peridinin-containing plastids" in the majority of photosynthetic dinoflagellates in both pigment composition and evolutionary origin. The plastids in members of genera Karenia and Karlodinium (family Kareniaceae) contain 19'-hexanoylfucoxanthin along with $\mathrm{Chl} a+c$, which are remnants of an endosymbiotic haptophyte (Bjørnland et al, 2003; Tengs et al, 2000; Zapata et al, 2012). Members of the genus Lepidodinium (family Gymnodiniaceae) established the current plastids containing chlorophylls $a$ and $b$ through the endosymbiosis of a pedinophyte green alga (Watanabe et al, 1987, 1990; Matsumoto et al, 2011; Kamikawa et al, 2015a). At the morphological level, the endosymbionts in the two lineages described above were extensively reduced in the host cells, leaving only plastids in the endosymbiont-derived compartments. At the genetic level, endosymbiont genes, particularly ones for plastid functions and maintenance (henceforth we designate as "plastid-related genes"), were transplanted into the host genomes of the two dinoflagellate lineages (Ishida \& Green 2002; Takishita et al, 2004; Nosenko et al, 2006; Patron et al, 2006; Minge et al, 2010; Burki et al, 2014). Such gene transfer from an endosymbiont to its host (endosymbiotic gene transfer or EGT) is regarded as one of the keys for the host-endosymbiont interlock at the genetic level. Thus, both haptophyte-derived and green alga-derived plastids in dinoflagellates are regarded as genuine organelles in the current host cells.

In addition to endosymbiotically transferred genes, Karenia (Ke.) brevis, Karlodinium (Kl.) veneficum, and Lepidodinium chlorophorum are known to possess plastid-related genes, which bear phylogenetic affinities to the orthologous genes in peridinin-containing dinoflagellates (Takishita et al, 2004, 2008; Minge et al, 2010; Nosenko et al, 2006; Patron et al, 2006; Burki et al, 2014; Bentlage et al, 2016). As the peridinin-containing plastid was most likely established in the ancestral dinoflagellates, it is reasonable to assume that the genes described above have been inherited vertically throughout the dinoflagellate evolution, rather than acquired from the 
endosymbiont. Furthermore, a certain fraction of plastid-related genes was unlikely to be vertically inherited from the ancestral dinoflagellates or endosymbiotically acquired from eukaryotic algae that gave rise to non-canonical plastids (Nosenko et al. 2006; Patron et al, 2006; Minge et al, 2010; Burki et al, 2014; Bentlage et al, 2016). In light of previous gene surveys in the Ke. brevis/Kl. veneficum and L. chlorophorum transcriptomic data, it is clear that the proteomes in the noncanonical plastids in these dinoflagellates are composed of the proteins with diverse evolutionary origins. However, to our knowledge, it has been unclear whether the overall degree of evolutionary "chimerism" in plastid proteome varies among non-canonical plastids established separately in dinoflagellate evolution. Likewise, we are uncertain whether there is an evolutionary consistent pattern in chimerism across multiple plastid-localized metabolic pathways within a non-canonical plastid. Prior to this study, the individual evolutionary origins of enzymes involved in the heme and isopentenyl diphosphate (IPP) biosynthetic pathways in diverse "complex algae," which acquired the plastids through green or red algal endosymbioses, have been characterized (Wilhelm et al, 2006; Cihláŕ \& Füssy et al, 2016; Bentlage et al, 2016), albeit no comparison among their overall patterns has been compared to each other. In this study, we investigate the evolutionary origins of enzymes involved in the biosyntheses of heme and IPP, as well as that of chlorophyll $a$ (Chl a), which links to the two aforementioned pathways, in Ke. brevis/Kl. veneficum and $L$. chlorophorum.

The vast majority of the enzymes involved in C5 pathway for the heme biosynthesis, the non-mevalonate pathway for IPP biosynthesis, and the Chl $a$ biosynthesis is nucleus-encoded in photosynthetic eukaryotes (Oborník \& Green, 2005; Bentlage et al, 2016). The three pathways in photosynthetic eukaryotes and those in cyanobacteria are principally homologous to each other (Hunter, 2007; Sousa et al, 2013; Oborník \& Green, 2005), suggesting that the pathways in photosynthetic eukaryotes can be traced back to those endosymbiotically acquired from the 
cyanobacterial endosymbiont that gave rise to the first plastid (primary plastid). Consistent with their cyanobacterial ancestry, the three pathways described above occur in the plastids in photosynthetic eukaryotes. In the following paragraphs, we briefly overview the three pathways. C5 pathway for the heme biosynthesis (Fig. 1) transforms glutamyl-tRNA to protoheme through 9 consecutive enzymatic steps, and the $7^{\text {th }}$ and $8^{\text {th }}$ steps are catalysed by functionally homologous but evolutionarily distinct enzymes (Fig. 1)-hemN and hemF for the coproporphyrinogen oxidase (CPOX) activity (Panek \& O'Brian, 2002), and hemJ and hemY for the protoporphyrinogen IX oxidase (PPOX) activity (Kobayashi et al, 2014). We are aware of the heme biosynthetic pathway in apicomplexan parasites and their relatives being diversified (Kořený et al, 2011). However, we do not mention these exceptions below, as we can discuss the evolutions of the heme biosynthesis in Ke. brevis/Kl. veneficum and L. chlorophorum without acknowledging the unorthodox pathways in apicomplexan parasites and their relatives.

As shown in Fig. 1, the pathway synthesizing Chl $a$ and C5 pathway for the heme biosynthesis share a common precursor, and the first to $8^{\text {th }}$ enzymatic steps converting glutamyltRNA to protoporphyrin IX (Reinbothe et al, 1996; Beale, 1999). We regard the steps converting protoporphyrin IX to $\mathrm{Chl} a$ as the "Chl $a$ biosynthetic pathway" in this study. As seen in the heme biosynthesis, two evolutionarily distinct enzymes are known to catalyze three out of the 6 steps (Fig. 1). Firstly, there are two evolutionarily distinct types of Mg-protoporphyrin IX monomethyl ester cyclase (MgPME cyclase), one is a multi-subunit enzyme (Yamanashi et al, 2015; Chen et al, 2017) and the other is a single-subunit enzyme encoded by chlE (Yamanashi et al, 2015). To our knowledge, neither of the enzymes has been detected in complex algae (e.g., diatoms; Wilhelm et al. 2006; Nymark et al. 2009). Secondly, two evolutionarily distinct types of divinyl chlorophyllide $a$ 8-vinyl-reductase (DVR), namely N-DVR and F-DVR, are known, and the former and the latter use NADPH and reduced ferredoxin for electron donors, respectively (Ito et al, 2008; 
133 Ito \& Tanaka, 2014). Thirdly, light-dependent and light-independent types of protochlorophyllide 134 reductase (POR) have been identified (Fujita et al, 2003). The light-dependent POR is a single 135 polypeptide and encoded by a nuclear gene, por. The light-independent version comprises three 136 hetero subunits encoded by $\operatorname{chlB}$, chlL and $\operatorname{chlN}$, and some/all of the three genes are plastid-

\section{2} encoded in photosynthetic eukaryotes.

The non-mevalonate pathway for the IPP biosynthesis comprises 7 consecutive enzymatic steps (Fig. 1). To our knowledge, no functionally homologous but evolutionarily distinct enzyme has been known in this pathway. IPP is an essential precursor for various terpenoids including the phytol residue in Chl $a$. Thus, in land plants, the non-mevalonate pathway appeared to supply IPP to the Chl $a$ synthesis, suggesting that the two pathways tightly couple in the plastids (Lichtenthaler et al, 1997; Dubey et al, 2003).

We here surveyed the transcripts encoding enzymes involved in three plastid-localized metabolic pathways in two kareniacean species (Ke. brevis and $K l$. veneficum) and $L$. chlorophorum, which bear haptophyte-derived and green alga-derived plastids, respectively. Individual proteins identified in this study were then subjected to phylogenetic analyses to evaluate how the three pathways were modified during the haptophyte/green algal endosymbiosis. Our systematic assessment revealed that the impact of EGT was different among the three pathways in the three species examined in this study. We here propose biological reasons why the impact of EGT varied among the three pathways in the dinoflagellates bearing non-canonical plastids.

\section{Materials and Methods}

RNA-seq analysis of $L$. chlorophorum

L. chlorophorum NIES-1868 has been maintained in the laboratory as described in Kamikawa et 
157 al. (2015a). Total RNA was isolated from the harvested cells with TRIzol (Invitrogen) according 158 to the manufacture's instruction. We sent the RNA sample to Hokkaido System Science (Sapporo, 159 Hokkaido, Japan) for library construction with Truseq RNA Sample Prep Kit (Illumina) followed 160 by sequencing on a Illumina HiSeq 2000 platform. We yielded $\sim 386$ million paired-end reads ( $\sim 39$

161

162

163 164 165 166 167 168 169 170

Mbp in total). After exclsion of poorly called reads and removal of an adaptor sequence by FASTX-toolkit (http://hannonlab.cshl.edu/fastx_toolkit/download.html), we finally assembled the curated reads into 77841 contigs by Trinity ver.2.1.0 (Haas et al, 2013). The RNA-seq data of $L$. chlorophorum was deposited in DDBJ Sequence Read Archive (DRA accession number DRA006544)

We evaluated the coverage of the contig data yielded from the L. chlorophorum RNA-seq data against the "universally conserved genes" in eukaryotes with BUSCO ver. 3 (Waterhouse et al, 2017). The proportions of "complete," "fragmented" and "missing" BUSCOs were 84.2, 3.3 and $12.5 \%$, respectively.

\section{Survey of the genes encoding proteins involved in the heme, Chl $a$ and IPP biosyntheses}

In this study, we conducted phylogenetic analyses on the proteins involved in "Porphyrin and chlorophyll metabolism" (map00860) and "Terpenoid backbone biosynthesis" (map00900) in Kyoto Encyclopedia of Genes and Genomes pathway (KEGG pathway, http://www.genome.jp/kegg/pathway.html). KOIDs of the proteins subjected to the investigation in this study were K02492, K01845, K01698, K01749, K01719, K01599, K00228, K02495, K00231, K01772, K03403, K03404, K03405, K03428, K04034, K04035, K04036, K00218, K04037, K04038, K04039, K01662, K00099, K00991, K00919, K01770, K03526 and K03527 (see also Fig .1). To generate amino acid sequence alignments covering phylogenetically diverse eukaryotic algae and prokaryotes, we surveyed the sequences of interest in the contigs generated 
181 from the RNA-seq data of L. chlorophorum (see above) and public databases including the Marine Microbial Eukaryote Transcriptome Sequencing Project $\quad$ (MMETSP;

183 http://marinemicroeukaryotes.org), which provides the contigs from the combined assemblies of 184 the Ke. brevis and Kl. veneficum RNA-seq data ("Karenia-brevis-Wilson," "Karenia-brevis185 or BLASTP. In the first BLAST search, multiple sequences registered in the KOIDs listed above were used as queries except the enzymes described below. Two evolutionarily distinct versions were known for PPOX ("hemY-type" and "hemJ-type"), and we used KOID K00231 as the query to identify the former type of PPOX. We surveyed hemJ-type PPOX sequences, which is not registered in the current KEGG pathway, by using UPF0093 membrane protein encoded by slr1790 in Synechocystis sp. strain PCC 6803 (UniProtKB P72793) as the query. Both N-DVR and F-DVR are included in a single KOID (K19073), and the two distinct types of DVR sequences were separately subjected to the initial survey as queries (Meguro et al, 2011; Nagata et al, 2005). The accession numbers for the queries used in the initial BLAST searches are summarized in Table $\mathrm{S} 1$. The candidate sequences matched with the queries with $E$-values smaller than $10^{-20}$ in the first BLAST searches were retained. These candidate (amino acid) sequences were then subjected to BLASTP searches against the NCBI nr database (threshold was set as $E$-value of $10^{-5}$ ). Based on the results of the second BLAST searches, we retrieved the sequences matched to the proteins involved in the heme, Chl $a$ and IPP biosyntheses.

We further subjected the Ke. brevis and Kl. veneficum sequences selected by the procedure described above to BLASTP against the entire MMETSP database (https://www.imicrobe.us/\#/projects/104) to examine potential cross-contamination among multiple RNA-seq data deposited in the database (Marron et al, 2016; Dorrell \& Gile et al, 2017). 
205 brevis or Kl. veneficum (data not shown). Thus, we conclude that the Ke. brevis and Kl. veneficum

206 sequences retrieved from the MMETSP database are highly unlikely contaminants from non207 kareniacean organisms.

208 The nucleotide sequences (and their detailed information) identified in this study are 209 summarized in Tables S2. We also deposited their conceptual amino acid sequence at an online 210 repository (https://drive.google.com/drive/folders/1X_CsF15twTbr211 ziKENyADEVDidSJBOB_?usp=sharing).

\section{Phylogenetic analyses}

214

In this study, we investigated the origins of individual proteins involved in the three pathways for the heme, Chl $a$ and IPP biosyntheses in Ke. brevis, Kl. veneficum and L. chlorophorum by phylogenetic analyses with the maximum-likelihood (ML) method. For each protein of interest, we aligned the amino acid sequences retrieved from the public sequence databases described in the previous section by MAFFT v7.149b (Katoh \& Standley, 2013) with the L-INS-i option. The accession numbers of the sequences included in the alignments were listed in Table S3. The resultant alignments were manually refined, followed by exclusion of ambiguously aligned positions (see Table S4 for the details). The final alignments were individually subjected to RAxML 8.0.20 (Stamatakis, 2014). The ML tree was selected from 10 heuristic tree searches, each of which was initiated from a randomized stepwise addition parsimony tree. The most appropriate amino acid substitution model was selected for each alignment by ProtTest 3.4 (Darriba et al, 2011). The substitution models applied to the ML phylogenetic analyses are summarized in Table S4. ML bootstrap values (MLBPs) were calculated by summarizing 100 trees, each of which was inferred from a bootstrap data by a single heuristic tree search (see above). Bayesian analyses were conducted with PhyloBayes 4.1c under the CAT-GTR model with a discrete $\Gamma$ distribution with 
229 four categories (Lartillot \& Philippe, 2004). Four independent MCMC chains were run in parallel.

230 The detailed settings of the PhyloBayes analyses are summarized in Table S4. The individual

231 alignments and Newick format treefiles inferred from both ML and Bayesian methods are available 232 in the online repository (see above).

233

234

235

236

237

238

239

240

241

242

243

244

245

246

247

248

249

250

251

252

\section{In silico examination of plastid-localizing potential of $\mathrm{N}$-terminal extensions}

Nucleus-encoded proteins localized to complex plastids usually bear "bipartite" plastid-localizing signals, which are composed of an endoplasmic reticulum-targeting signal peptide (SP) and transit peptide-like sequence at their N-termini (Bolte et al, 2009). Firstly, we identified extra amino acid residues at their $\mathrm{N}$-termini (N-terminal extensions) in the putative Ke. brevis/Kl. veneficum and $L$. chlorophorum proteins involved in the heme, Chl $a$ and IPP biosyntheses by comparing the corresponding bacterial homologues (most of them are from cyanobacteria). We subsequently searched the SP in each N-terminal extension by SignalP v.4.1 (Petersen et al. 2011). In case of the SP being predicted, the transit peptide (TP) potential in the non-SP portions of the N-terminal extension was examined by ChloroP v.1.1 (Emanuelsson et al, 1999). SignalP and ChloroP analyses were done with the default settings. The results from the analyses on the N-terminal extensions can be found in Table S5.

\section{Results}

Henceforth here, we designated the proteins inherited from the ancestral dinoflagellate as "vertically inherited-type" or "VI-type," (ii) those acquired from an endosymbiont (i.e. haptophyte and green alga, in Ke. brevis/Kl. veneficum and L. chlorophorum, respectively) as "endosymbiotically acquired-type" or "EA-type," and (iii) those acquired from organisms 
253 distantly related to the host or endosymbiont lineages as "laterally acquired-type" or "LA-type." 254 A certain fraction of the proteins investigated could not be categorized into any of the three types 255 described above due to the lack of phylogenetic signal in alignments (see below).

256

257

258

259

260

261

262

263

264

265

266

267

268

269

270

271

272

273

274 275

276

\section{Heme biosynthetic pathway}

We successfully identified all or most of the enzymes required for the heme biosynthesis in the transcriptomic data of Ke. brevis, Kl. veneficum and L. chlorophorum. In Ke. brevis and Kl. veneficum, 8 out of the 9 enzymes were identified — only uroporphyrinogen III synthase (UROS) and coproporphyrinogen oxidase (CPOX) were missed in the former and latter, respectively. It is most likely that the UROS/CPOX transcripts were simply missed from the Ke. brevis/Kl. veneficum cDNA libraries, as UROS is essential to convert hydroxymethylbilane to uroporphyrinogen III, and CPOX is indispensable to yield protoporphyrinogen IX from coproporphyrinogen III. Fortunately, the Ke. brevis UROS sequence has already deposited in the GenBank database under the accession number CO063310, and was phylogenetically analyzed in this study (see below). Fifty-four out of the 60 transcripts (those encoding putative cytosolic proteins were excluded; see below) investigated here were found to bear N-terminal extensions. The SP were predicted in 21 out of the $54 \mathrm{~N}$-terminal extensions. Seven N-terminal extensions were predicted to bear both SP and TP. The details of the N-terminal extensions are summarized in Table S5.

Proteins with evolutionarily diverse origins comprise the Ke. brevis and Kl. veneficum pathways

To convert glutamyl-tRNA to glutamate-1-semialdehyde, Ke. brevis and Kl. veneficum possesses 
277 two versions of glutamyl-tRNA reductase (GTR). We consider "Karenia-1" and "Karlodinium-1"

278 sequences as VI-type, as they were nested within a clade with those of peridinin-containing

279 dinoflagellates, and the clade as a whole received full statistical support in the GTR phylogeny

280 (Fig. 2A). On the other hand, the second version of GTR in the two kareniacean species (Karenia-2

281 and Karlodinium-2) were most likely acquired from the haptophyte endosymbiont (i.e. EA-type).

282 The two GTR sequences were nested within the haptophyte clade, and the haptophyte clade

283 (including the Karenia-2 and Karlodinium-2 sequences) was supported by a MLBP of $66 \%$ and a

284 BPP of 0.99 (Fig. 2A).

Aminolevulinic acid (ALA) is synthesized from glutamate-1-semialdehyde by glutamate1-semialdehyde 2,1-aminomutase (GSAT). We identified a single version of GSAT in both $\mathrm{Ke}$. brevis and $\mathrm{Kl}$. veneficum. The possibility of the two GSAT sequences being acquired from the haptophyte endosymbiont can be excluded, as the haptophyte sequences (except that of Pavlova sp.) formed a robust clade and excluded the kareniacean sequences in the GSAT phylogeny (Fig. 2B). Nevertheless, the phylogenetic origin of either Kl. veneficum or Ke. brevis GSAT sequence could not be pinpointed any further. The GSAT sequences of Ke. brevis and peridinin-containing dinoflagellates grouped together in the both ML and Bayesian phylogenies, but their monophyly was poorly supported (MLBP of $8 \%$ and BPP $<0.50$ ). The $K l$. veneficum GSAT sequence showed no specific affinity to any sequence examined here. Thus, we withhold to conclude the precise origins of the GSAT sequences of Ke. brevis and Kl. veneficum in this study. acid dehydratase (ALAD) homologue in both Ke. brevis and Kl. veneficum. Both Ke. brevis and Kl. veneficum ALAD sequences formed a robustly supported clade with those of peridinincontaining dinoflagellates (MLBP of $99 \%$ and BPP of 1.0; Fig. 2C), suggesting that the two sequences are of VI-type. 

porphobilinogen to synthesize hydroxymethylbilane, in Ke. brevis (Karenia-1 and 2). The PBGD phylogeny (Fig. 2D) recovered (i) a clade of the sequences of peridinin-containing dinoflagellates and Karenia-1 sequence with a MLBP of $100 \%$ and a BPP of 0.99 , and (ii) a clade of the haptophyte and Karenia-2 sequences with a MLBP of $73 \%$ and a BPP of 0.83 . Thus, Ke. brevis seemingly uses both VI-type and EA-type enzymes for hydroxymethylbilane synthesis. We identified four PBGD sequences in Kl. veneficum (Karlodinium-1-4), which clearly share a single ancestral sequence. The $K l$. veneficum clade appeared to be distant from the clade comprising the sequences of peridinin-containing dinoflagellates and the Karenia-1 sequence, suggesting that the Karlodinium-1-4 sequences are not of VI-type. The Kl. veneficum clade was connected to the haptophyte clade (including the Karenia-2 sequence) with a MLBP of 47\% and a BPP of 0.67 (highlighted by an arrowhead in Fig. 2D). The statistical support for the particular node is insufficient to conclude or exclude the haptophyte origin of the Karlodinium sequences with confidence. Thus, we determined to leave the origin of the Karlodinium sequences uncertain. We assessed the evolutionary origins of two distinct versions of UROS in $\mathrm{Kl}$. veneficum (Karlodinium-1 and 2), and a single UROS sequence detected in an expressed-sequence tag data from Ke. brevis (Fig. 2E). The Karenia sequence, Karlodinium-1 and peridinin-containing dinoflagellate sequences grouped together with a MLBP of $76 \%$ and BPP of 0.95 . Thus, the Karenia and Karlodinium-1 sequences are most likely of VI-type. The Karlodinium-2 appeared to be remote from the dinoflagellate or haptophyte clade, suggesting this sequence was of LA-type (Fig. 2E). The UROS phylogeny connected the Karlodinium-2 sequence and that of a red alga Rhodosorus marinus with a MLBP of 95\% and BPP of 0.93, and the "Karlodinium + Rhodosorus" 323 clade was nested within the bacterial sequences. The particular clade could have been generated 324 by two separate gene transfers from a single bacterium (or two closely related bacteria) to $\mathrm{Kl}$. 
325

326

327

328

329

330

331

332

333

334

335

336

337

338

339

340

341

342

343

344

345

346

347

348

veneficum and Rhodosorus. Alternatively, the combination of the first gene transfer from a bacterium to either $\mathrm{Kl}$. veneficum or Rhodosorus, and the second one between the two eukaryotes may have produced the "Karlodinium + Rhodosorus" clade in the UROS phylogeny. In either of the two scenarios, we can regard the Karlodinium-2 sequence as LA-type. We need to increase the number of red algal UROS sequences in the future studies to retrace the precise origins of the Karlodinium and Rhodosorus sequences.

Four acetyl side chains in uroporphyrinogen III are removed by uroporphyrinogen decarboxylase (UROD) to generate coproporphyrinogen III. Pioneering studies revealed that photosynthetic eukaryotes with complex plastids possess evolutionarily distinct, multiple versions of UROD (Kořený et al, 2011; Cihlář \& Füssy et al, 2016). The UROD sequences of peridinincontaining dinoflagellates were split into three clades in the UROD phylogeny (designated as D1, D2 and D3 clades in Fig. 2F), suggesting that the three distinct versions have already been established in the ancestral dinoflagellate. Likewise, haptophytes were found to possess three distinct versions of UROD (designated as H1, H2 and $\mathrm{H} 3$ clades in Fig. 2F). We here identified five and four UROD sequences in Ke. brevis and Kl. veneficum, respectively. Among the five sequences identified in Ke. brevis, the "Karenia-1, 2 and 4" sequences were considered as EAtype, as they were placed within the haptophyte sequences in the UROD phylogeny (Fig. 2F). H1 clade including the Karenia-1 and 2 sequences received a MLBP of 100\% and a BPP 1.0. The Karenia-4 sequence and the haptophyte sequences (except that of Pavlova) formed H3 clade, of which monophyly was supported by a MLBP of $79 \%$ and a BPP of 0.99 . The "Karenia-3" sequence grouped with the sequence of a euglenid Eutreptiella gymnastica with a MLBP of 100\% and a BPP of 0.99. As the Karenia-3 sequence showed no affinity to the dinoflagellate or haptophyte clade in the UROD phylogeny, we categorize it in LA-type. The intimate phylogenetic affinity between the Karenia-3 and E. gymnastica sequences hints either (i) gene transfer between 
349 the two organisms or (ii) two separate gene transfers from an as-yet-unknown organism to $\mathrm{Ke}$.

350 brevis and E. gymnastica. It is also important to improve the sequence sampling from euglenids in

351 future phylogenetic studies to understand precisely the biological event generated the union of the

352 Karenia-3 and E. gymnastica sequences. The "Karenia-5" sequence is most likely descended from

353 one of the UROD versions established in the ancestral dinoflagellate (i.e. VI-type), as this sequence

354 participated in D3 clade, of which monophyly received a MLBP of 100\% and a BPP of 1.0. We

355 also assessed the origins of four Kl. veneficum sequences (Karlodinium-1-4) based on the UROD

356 phylogeny (Fig. 2F). The Karlodinium-1 and 2 sequences were nested within H1 clade, and the

357 Karlodinium-4 sequence were placed within H3 clade. Thus, we conclude that the three UROD

358 sequences are of EA-type. The Karlodinium-3, one of the L. chlorophorum sequences (see below),

359 and diatom sequences formed a robust clade (MLBP of 100\% and BPP of 1.0; highlighted by an

360 arrowhead in Fig. 2F), suggesting that the Karlodinium-3 sequence were laterally acquired from a

361 diatom (i.e. LA-type).

362 Coproporphyrinogen III is oxidized by CPOX to yield protoporphyrinogen IX. We 363 identified a single version of CPOX in Ke. brevis, but none in Kl. veneficum. The CPOX phylogeny 364 (Fig. 2G) recovered a clade comprising the sequences of peridinin-containing dinoflagellates and 365 Ke. Brevis with a MLBP of $98 \%$ and a BPP of 0.99 . Thus, Ke. brevis uses a VI-type CPOX. obtain protoporphyrin IX. The PPOX sequences of peridinin-containing dinoflagellates were separated into two distinct clades labeled as "D1" and "D2" (Fig. 2H), and both received MLBPs of $100 \%$ and BPPs $\geq 0.97$. The PPOX sequences in D2 clade are likely cytosolic version, as these dinoflagellate sequences, as well as those of other photosynthetic eukaryotes bearing complex 371 plastids (chlorarachniophytes, Euglena gracilis and Vitrella brassicaformis), formed a robust clade 372 with the PPOX sequences of heterotrophic eukaryotes (MLBP of 93\% and BPP of 0.99 ; 
373 highlighted by an arrowhead in Fig. $2 \mathrm{H}$ ). We identified two versions of PPOX in Ke. brevis

374 (Karenia-1 and 2), while a single version was identified in Kl. veneficum. The Karenia-1 sequence

375 fell into D1 clade (Fig. 2H), suggesting that this PPOX sequence is of VI-type. On the other hand, 376 the PPOX phylogeny united the Karenia-2 and a single PPOX of Kl. veneficum with the sequences 377 of stramenopiles, haptophytes and L. chlorophorum with a MLBP of $100 \%$ and a BPP of 0.99 378 (highlighted by a double-arrowhead in Fig. 2H). As the bipartitions within the clade were 379 principally unresolved (Fig. 2H), we cannot exclude the possibility of the Karenia-2 and 380 Karlodinium-1 sequences group with the haptophyte sequences in this clade. The Karenia-2 and

Karlodinium-1 sequences are definitely not of VI-type, but the phylogenetic resolution was not sufficient to classify the two sequences into of EA-type or LA-type. Thus, we decide to leave the origins of the Karenia-2 and Karlodinium-1 sequences uncertain.

In the last step in the heme biosynthesis, ferrochelatase $(\mathrm{FeCH})$ converts protoporphyrin IX to protoheme. None of the $\mathrm{FeCH}$ sequences identified in Ke. brevis and $\mathrm{Kl}$. veneficum appeared to be of VI-type. Ke. brevis possesses three distinct versions of FeCH (Karenia-1-3). The FeCH phylogeny (Fig. 2I) united the Karenia-1 sequence with one of four versions of FeCH in $L$. chlorophorum (Lepidodinium-3) with a MLBP of 100\% and a BPP of 1.0, and this union was then connected specifically to $\gamma$-proteobacterial sequences with a MLBP of $75 \%$ and a BPP of 0.99 (highlighted by an arrowhead in Fig. 2I). This subtree can be explained by two sequential gene transfer events, namely the first gene transfer from a $\gamma$-proteobacterium to either L. chlorophorum or Ke. brevis, and the second one between the two dinoflagellates. Consequently, the Karenia-1 and Lepidodinium-3 sequences can be of LA-type, and traced back to the bacterial sequence. The Karenia-2 sequence is unlikely to be of VI-type, as the sequences of peridinin-containing dinoflagellates (and one of the sequences of L. chlorophorum) were united with a MLBP of 100\% and a BPP of 1.0 (Fig. 2I). The Karenia-2 sequence, which showed no clear affinity to the 
397

398

399

400

401

402

403

404

405

406

407

408

409

410

411

412

413

414

415

416

417

418

419

420

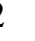

haptophyte sequences, is unlikely to be of EA-type (Fig. 2I). Thus, we conclude that the Karenia-2 sequence is of LA-type, although the precise donor remains uncertain. Finally, the $\mathrm{FeCH}$ phylogeny grouped the Karenia-3 sequence and a single $\mathrm{FeCH}$ sequence identified in $K l$. veneficum with the cyanobacterial sequences, and their monophyly was supported by a MLBP of $76 \%$ and a BPP of 0.94 (highlighted by a double-arrowhead in Fig. 2I). We conclude that the two dinoflagellate sequences are of cyanobacterial origin (i.e. LA-type).

Pioneering studies on kareniacean dinoflagellates revealed the evolutionary chimeric natures of the plastid proteomes of Ke. brevis and Kl. veneficum, which comprises VI-, EA- and LA-type proteins (Ishida \& Green 2002; Nosenko et al., 2006; Patron et al., 2006; Burki et al., 2014). This study further enables us to evaluate the precise contribution of EA-type proteins on the evolution of the heme biosynthetic pathway in kareniacean dinoflagellates - EA-type proteins found only in three out of the 9 steps, namely (i) GTR in Ke. brevis and Kl. veneficum, (ii) PBGD in Kl. veneficum, and (iii) UROD in Ke. brevis and Kl. veneficum.

Little impact of endosymbiotic gene transfer on the L. chlorophorum pathway

We identified three versions of GTR in L. chlorophorum (Lepidodinium-1-3). The Lepidodinium-1 sequence grouped with the sequence of peridinin-containing dinoflagellates (and the Karenia-1 and Karlodinium-1 sequences), and this "dinoflagellate" clade received a MLBP of $100 \%$ and a BPP of 1.0 (Fig. 2A). Thus, we conclude that the Lepidodinium-1 sequence is of VItype. The Lepidodinium-2 and 3 sequences were tied to each other with a MLBP of $100 \%$ and a BPP of 1.0, and this clade was connected with the "dinoflagellate" clade described above (Fig. 2A). However, the phylogenetic affinity between the two clades received little statistical support (MLBP of 38\% and BPP $<0.50$; highlighted by an arrowhead in Fig. 2A), suggesting that the 
421 Lepidodinium-2 and 3 sequences were unlikely of VI-type. The GTR sequences of land plants and 422 green algae (plus a euglenid Eutreptiella gymnastica) formed a clade supported by a MLBP of $423100 \%$ and a BPP of 0.99 , and appeared to be distant from the two sequences of L. chlorophorum 424 (Fig. 2A). Thus, we can exclude the green algal (endosymbiont) origin of the Lepidodinium-2 and 4253 sequences. Combined, the two sequences are regarded as LA-type, although the donor of the GTR gene to L. chlorophorum remains unclear.

428

430

431

432

433

434

435

436

We failed to classify two out of the three versions of GSAT identified in L. chlorophorum. In the GSAT phylogeny (Fig. 2B), the "Lepidodinium-1" and "Lepidodinium-2" sequences fell separately into the cluster of the sequences of peridinin-containing dinoflagellates and Ke. brevis, but this clade as a whole received no significant statistical support. Thus, we left the origins of the Lepidodinium-1 and 2 sequences uncertain in this study. On the other hand, the "Lepidodinium-3" sequence was excluded from the clade comprising the sequences of diverse photosynthetic eukaryotes and cyanobacteria with a MLBP of $100 \%$ and a BPP of 1.0 , and connected to the sequences of Streptomyces coelicolor, Mycobacterium tuberculosis and Corynebacterium diphtheriae with a MLBP of 71\% and a BPP of 0.91 (highlighted by an arrowhead in Fig. 2B). This tree topology suggests that the Lepidodinium-3 sequence was acquired from a bacterium (i.e. LA-type), albeit we cannot pinpoint the bacterium donated a GSAT gene to L. chlorophorum. Two versions of ALAD were identified in L. chlorophorum (Lepidodinium-1 and 2). The sequences of peridinin-containing dinoflagellates, the Lepidodinium-1 sequence and the sequences of Ke. brevis and Kl. veneficum clustered with a MLBP of $99 \%$ and a BPP of 1.0 in the ALAD phylogeny (Fig. 2C). Thus, we conclude the Lepidodinium-1 sequence as VI-type. The Lepidodinium-2 sequence was distantly related to the sequences of peridinin-containing dinoflagellate or green algae/land plants, but showed no strong affinity to any clade/sequence in the ALAD phylogeny (Fig. 2C). Thus, we propose the Lepidodinium-2 sequence as LA-type, albeit 
445 its precise origin was unresolved in the ALAD phylogeny.

446

447

448

449

450

451

452

453

454

455

456

457

458

459

460

461

462

Two versions of PBGD and a single version of UROS identified from L. chlorophorum.

The PBGD phylogeny (Fig. 2D) united the two sequences of L. chlorophorum together with a MLBP of $100 \%$ and a BPP of 0.99 , but this clade showed little phylogenetic affinity to the sequences of peridinin-containing dinoflagellates, green algae/land plants or any sequences considered in the phylogenetic analysis. Likewise, the UROS phylogeny (Fig. 2E) recovered no clear affinity of the sequence of $L$. chlorophorum to other sequences including those of peridinincontaining dinoflagellates or green algae/land plants. Thus, L. chlorophorum likely uses LA-type PBGD and UROS, but their precise origins remain uncertain.

We identified 7 versions of UROD in L. chlorophorum, and 6 of them showed clear affinities to the sequences of peridinin-containing dinoflagellates. In the UROD phylogeny (Fig. 2F), the sequences of peridinin-containing dinoflagellates formed three distinct clades (D1-3 clades), and each of these clades enclosed at least one sequence of L. chlorophorum, namely (i) the "Lepidodinium-2" sequence in D1 clade, (ii) "Lepidodinium-3 and 4" sequences in D2 clade, and (iii) "Lepidodinium-5, 6 and 7" sequences in D3 clade. Thus, the 6 sequences described above are concluded as VI-type. The "Lepidodinium-1" sequence and sequences of diatoms (and $K l$. veneficum) formed a clade with a MLBP of $100 \%$ and a BPP of 1.0 (highlighted by an arrowhead in Fig. 2F), suggesting that this version was acquired from a diatom (i.e. LA-type).

Three versions of CPOX were identified in L. chlorophorum, but none of them was of VI-type or EA-type. The CPOX phylogeny (Fig. 2F) placed the "Lepidodinium-1" sequence in a remote position from the sequences of peridinin-containing dinoflagellates or green algae/land plants. Instead, the Lepidodinium-1 sequence grouped with the bacterial sequences, as well as the eukaryotic sequences for the cytosolic pathway, with a MLBP of $77 \%$ and a BPP of 0.87 (highlighted by an arrowhead in Fig. 2G). This protein most likely bears no N-terminal extension 
469 (Table S5). Altogether, we propose that the Lepidodinium-1 sequence encodes a cytosolic CPOX

470

471

472

473

474

475

476

477

478

479

480

481

482

483

484

485

486

487 enzyme involved in C4 pathway, and omitted from the discussion below. The "Lepidodinium-2" and "Lepidodinium-3" sequences share high sequence similarity in the mature protein region, while their N-terminal regions are distinct from each other (Table S5). In the CPOX phylogeny, the two Lepidodinium sequences showed a specific affinity to the diatom sequences (MLBP of 86\% and BPP of 0.98 ; highlighted by a double-arrowhead in Fig. $2 \mathrm{G})$, instead of the dinoflagellate or green plant sequences. Thus, the ancestral CPOX of the two L. chlorophorum sequences was most likely of LA-type.

We conclude that L. chlorophorum possesses two distinct VI-type (Lepidodinium-1 and 3) and a single LA-type PPOX (Lepidodinium-2). The PPOX sequences of peridinin-containing dinoflagellates were split into two distinct clades (D1 and D2), and the two clades received strong statistical support from both ML bootstrap and Bayesian analyses (Fig. 2H). The Lepidodinium-1 and 3 sequences were included in D1 and D2 clades, respectively. As the PPOX sequences (including the Lepidodinium-3 sequence) in D2 clade can be considered as the cytosolic version, we did not discuss the Lepidodinium-3 sequence further. The PPOX phylogeny recovered a robust clade comprising the Lepidodinium-2 sequence and, the sequences of haptophytes, stramenopiles, Kl. veneficum and Ke. brevis (MLBP of $100 \%$ and BPP of 0.99 ; highlighted by a double-arrowhead in Fig. 2H). Thus, the Lepidodinium-2 sequence was acquired from an organism distantly related to dinoflagellates or green algae/land plants (i.e. LA-type).

In L. chlorophorum, we identified four versions of FeCH. The "Lepidodinium-1" sequence is of VI-type, as this sequence apparently shared the origin with the sequences of peridinin-containing dinoflagellates, and their monophyly was supported with a MLBP of 100\% and a BPP of 1.0 (Fig. 2I). On the other hand, we consider the rest of the sequences of $L$. chlorophorum as LA-type, as the "Lepidodinium-2," "Lepidodinium-3" and "Lepidodinium-4" 
493

494

495

496

497

498

499

500

501

502

503

504

505

506

507

508

509

510

511

512

513

514

515

516

sequences appeared to be distantly related to the dinoflagellate clade described above or the green algae/land plant sequences in the $\mathrm{FeCH}$ phylogeny (Fig. 2I). The precise positions of the Lepidodinium-2 and Lepidodinium-4 sequences were unresolved, and it remains unclear how $L$. chlorophorum acquired the two versions of $\mathrm{FeCH}$. On the other hand, the $\mathrm{FeCH}$ phylogeny united the "Lepidodinium-3" and Karenia-1 sequences together (MLBP of 100\% and BPP of 1.0), and this dinoflagellate clade was then connected to two $\gamma$-proteobacterial sequences with a MLBP of $75 \%$ and a BPP of 0.99 (highlighted by an arrowhead in Fig. 2I). We have already proposed the two scenarios for the origin of the Lepidodinium-3 and Karenia-1 sequences, in which two lateral gene transfers were invoked (see the previous section for the details).

The most prominent feature in the L. chlorophorum pathway is that no gene from the green algal endosymbiont was detected in heme biosynthesis. Instead, genes transferred from organisms related to neither host (dinoflagellate) nor endosymbiont (green alga) largely contributed to the L. chlorophorum pathway. The impact of lateral gene transfer on the heme biosynthesis is most prominent in the steps catalyzed by PBGD, UROS and CPOX, in which only LA-type proteins were identified.

Chl $a$ biosynthetic pathway

We surveyed the transcripts encoding enzymes involved in the Chl a biosynthesis in Ke. brevis, Kl. veneficum and L. chlorophorum, and assessed their origins individually. Overall, all the enzymes required to synthesize $\mathrm{Chl} a$, except $\mathrm{MgPME}$ cyclase, was retrieved from the transcriptomic data from the three dinoflagellates. To our knowledge, no sign for nucleus-encoded MgPME cyclase, which converts Mg-protoporphyrin IX monomethyl ester to divinyl protochlorophyllide, has been detected in complex algae (Wilhelm et al. 2006; Nymark et al. 
517 2009). Although not described in detail, we additionally surveyed the single-subunit MgPME 518 cyclase encoded by chlE, which are phylogenetically distinct from the multi-subunit MgPME 519 cyclase, but yielded no significant match. We suspect an as-yet-unidentified enzyme forming E520 ring in the photosynthetic eukaryotes described above. Twenty-two out of the 27 transcripts 521 investigated here were found to possess N-terminal extensions, which likely work as plastid522 localizing signals (Note that 11 sequences were proceeded by the putative SPs, and two of their non-SP portion were predicted to have the TP potential; see Table S5 for the details).

Large impact of EGT on the Ke. brevis and Kl. veneficum pathways

$\mathrm{Mg}$ chelatase $(\mathrm{MgCH})$, which comprises three subunits $\mathrm{ChlD}, \mathrm{ChlH}$ and ChlI, inserts $\mathrm{Mg}^{2+}$ to protoporphyrin IX. In Kl. veneficum, ChlI is plastid-encoded (Gabrielsen et al. 2011) and the rest of the subunits were nucleus-encoded (see below). Although no plastid genome data is available for Ke. brevis, chlI is predicted to reside in the plastid genome of Karenia mikimotoi based on a pioneering study on the plastid transcriptome of this species (Dorrell et al, 2016). In this study, we identified the partial chlI contig in the transcriptomic data of Ke. brevis (contig No. 0173787962). The 5' terminus of this contig was not completed, we could not conclude whether the chlI transcript received the poly $(\mathrm{U})$ tail, which is the unique RNA modification occurred in peridinin-containing plastids as well as the non-canonical plastids of Ke. mikimoti and Kl. veneficum (Dorrell \& Howe, 2012; Richardson et al, 2014). Although the precise genome harboring chlI in Ke. brevis remains uncertain, we assume that the Ke. brevis chlI sequence was transcribed from the plastid genome as demonstrated in Ke. mikimotoi by Richardson et al. (2014), although no plastid genome is available. As the current study focuses on nucleus-encoded proteins involved in plastid 540 metabolisms, we stopped examining the origin and evolution of ChlI in the two kareniacean 
541 species (and L. chlorophorum; see below) any further.

We here examine the evolutionary origins of two nucleus-encoded subunits of $\mathrm{MgCH}$,

543

544

$\mathrm{ChlH}$ and ChlD, in Ke. brevis and Kl. veneficum. A single version of ChlD was identified in Ke. brevis and $\mathrm{Kl}$. veneficum. The ChlD phylogeny (Fig. 3A) recovered a clade of the sequences of haptophytes, Ke. brevis and Kl. veneficum with full statistical support, suggesting that the kareniacean ChlD sequences are of EA-type. Both Ke. brevis and Kl. veneficum possess two distinct versions of $\mathrm{ChlH}$ (Karenia-1 and 2, and Karlodinium-1 and 2). ChlH sequences can be split in to two distinct clades, "ChlH-1" and "ChlH-2," as a previous study reported (Lohr et al, 2005, Fig. 3B). ChlH-1 sequences are ubiquitously distributed in photosynthetic organisms, while ChlH-2 sequences have been found in restricted lineages. The sequences of green algae/land plants formed two distinct clades (Gp1 and 2 clades). In the ChlH phylogeny (Fig. 3B), the haptophyte ChlH-1 sequences, the Karenia-1 and Karlodinium-1 sequences formed a clade supported with a MLBP of $98 \%$ and a BPP of 1.0. In contrast, the Karenia-2 and Karlodinium-2 sequences were nested within the Gp2 clade containing $\mathrm{ChlH}-2$ sequences of green algae and a euglenid, and their monophyly received a MLBP of $81 \%$ and a BPP of 0.98 (highlighted by an arrowhead in Fig. 3B). Thus, we conclude that Ke. brevis and Kl. veneficum possess $\mathrm{ChlH}-1$ sequences acquired from the haptophyte endosymbiont (i.e. EA-type), while their ChlH-2 sequences are of green algal origin (i.e. LA-type).

$S$-adenosylmethionine:Mg-protoporphyrin $O$-methyltransferase (MgPMT) converts $\mathrm{Mg}$ protoporphyrin IX to Mg protoporphyrin IX monomethyl ester. The MgPMT sequences of Ke. brevis and $\mathrm{Kl}$. veneficum grouped with the haptophyte sequences (except the one of Pavlova), and their monophyly was supported by a MLBP of $67 \%$ and a BPP of 0.99 (highlighted by an arrowhead in Fig. 3C). The two kareniacean species most likely use the MgPMT acquired from the haptophyte endosymbiont (i.e. EA-type). 
No MgPME cyclase has been identified in any dinoflagellates regardless of plastid-type,

566

567

568

and we could not examine the origin and evolution of this enzyme (see above). However, DVR, which recognizes the product of MgPME cyclase (divinyl protochlorophyllide) as the substrate and generate protochlorophyllide, were identified in both dinoflagellates bearing peridinin and those bearing non-canonical plastids. We identified both N-DVR and F-DVR sequences in $K e$. brevis, while only N-DVR sequence was found in Kl. veneficum. The F-DVR phylogeny (Fig. 3D) recovered the clade of the sequences of Ke. brevis and haptophytes with robust statistical support, suggesting that Ke. brevis was acquired from the haptophyte endosymbiont (i.e. EA-type).

The N-DVR phylogeny (Fig. 3E) united the sequences of Ke. brevis and Kl. veneficum together with a MLBP of $98 \%$ and a BPP of 0.99 , and the kareniacean clade showed any phylogenetic affinity to neither haptophyte sequences nor other dinoflagellate sequences. Instead, the kareniacean clade grouped with the sequences of stramenopiles, haptophytes, and two chromerids (Chromera and Vitrella) supported with a MLBP of $100 \%$ and a BPP of 1.0 (highlighted by an arrowhead in Fig. 3E). In this large clade, the affinity between the kareniacean clade and haptophyte sequences remained uncertain, and we are not sure of the precise origin of the two kareniacean sequences.

In land plants, conversion of protochlorophyllide to chlorophyllide $a$ is catalyzed by the light-dependent and/or light-independent forms of POR. The light-dependent POR is nucleusencoded, while the light-independent form comprises three plastid-encoded subunits (ChlB, ChlL and ChlN). No gene for light-independent POR was found in the sequenced region of the $K l$. veneficum plastid genome (Gabrielsen et al. 2011) or plastid transcriptomic data of Ke. mikimotoi (Dorrell et al. 2016), implying that kareniacean species lack the light-independent version. We identified two and three distinct versions of the light-dependent POR in Ke. brevis and Kl. veneficum, respectively (Karenia-1 and 2, and Karlodinium-1-3), as demonstrated in Hunsperger 
612 Genetic influx from phylogenetically diverse organisms shaped the L. chlorophorum pathway

et al, (2015). In the POR phylogeny (Fig. 3F), the Karenia-1 and Karlodinium-1 sequences grouped with the sequences of stramenopiles, cryptophytes and haptophytes, and their monophyly was supported by a MLBP of 95\% and a BPP of 0.98 (highlighted by an arrowhead in Fig. 3F). The Karenia-1 and Karlodinium-1 sequences cannot be of VI-type, as the two sequences are distantly related to other dinoflagellate sequences included in the alignment. However, it is difficult to classify the Karenia-1 and Karlodinium-1 sequences into EA-type or LA-type, as the relationship between the two dinoflagellate sequences and the haptophyte sequences was unresolved in the particular clade. Thus, we leave the origins of the two sequences uncertain in this study. The Karenia-2, Karlodinium-2 and Karlodinium-3 sequences robustly grouped together within the haptophyte sequences, and this "haptophyte" clade received a MLBP of 98\% and a BPP of 0.99 (Fig. 3F). Thus, we conclude that these sequences were acquired from the haptophyte endosymbiont (i.e. EA-type).

The final step of the Chl $a$ biosynthesis is catalyzed by Chl synthase (CS). A single version of CS was identified in Ke. brevis and Kl. veneficum. The two kareniacean sequences were placed within the haptophyte clade in the CS phylogeny, and the "haptophyte" clade as a whole was supported by a MLBP of $98 \%$ and a BPP of 1.0 (Fig. 3G). Thus, the sequences of Ke. brevis and Kl. veneficum are considered as EA-type.

The phylogenetic analyses described above revealed that EA-type proteins operate in all the steps converting protoporphyrin IX to $\mathrm{Chl} a$ in $K l$. veneficum and/or Ke. brevis (except the step catalyzed by MgPME cyclase; see above). In addition, the common ancestor of Ke. brevis and $\mathrm{Kl}$. veneficum should have possessed LA-type ChlH-2, POR and N-DVR, which were acquired from phylogenetically diverse eukaryotes distantly related to dinoflagellates or haptophytes.

611 
614 As discussed in the previous section, the Chl a biosynthetic pathway in Ke. brevis and Kl. 615 veneficum appeared to be shaped by the genes transferred from the endosymbiont (i.e. a haptophyte 616 in the above systems). Curiously, this is not the case for the same pathway in L. chlorophorum, of 617 which plastid was derived from a pedinophyte green alga. Note that we present no result from the 618 L. chlorophorum ChlI, which turned out to be plastid-encoded (Kamikawa et al., 2015a). We 619 identified 8 proteins involved in the Chl $a$ biosynthetic pathway in L. chlorophorum, and assess 620 their phylogenetic origins individually (Figs. 3A-G). Among the 8 proteins examined here, we conclude MgPMT as a sole EA-type protein among those involved in the L. chlorophorum pathway. The MgPMT phylogeny (Fig. 3C) placed the L. chlorophorum sequence within a radiation of the sequences of green algae, land plants and chlorarachniophytes, and their monophyly was supported by a MLBP of $88 \%$ and a BPP of 0.98 .

Our surveys and phylogenetic analyses revealed that VI-type proteins were almost entirely eliminated from the L. chlorophorum pathway. We identified only one of the two versions of POR (Lepidodinium-1) as VI-type. The POR phylogeny (Fig. 3F) recovered two distinct clades of the sequences of peridinin-containing dinoflagellates (D1 and D2 clades), and placed the Lepidodinium-1 sequence within D1 clade. D1 clade containing the Lepidodinium-1 sequence as a whole received a MLBP of $97 \%$ and a BPP of 0.99 .

We could not clarify the origin of the N-DVR sequence of L. chlorophorum, of which 632 position was resolved in neither ML nor Bayesian phylogenetic analyses (Fig. 3E). The 633 Lepidodinium sequence was excluded from the robust clade of the sequences of peridinincontaining dinoflagellates, suggesting that this sequence cannot be of VI-type. However, it is difficult to pursue the origin of the Lepidodinium sequence any further, as the N-DVR phylogeny 
637

638

639

640

641

642

643

644

645

646

647

648

649

650

651

652

653

654

655

656

657

658

659

660

sequences (Fig. 3E).

We classified ChlD, ChlH, one of the two versions of POR (Lepidodinium-2), F-DVR and CS into LA-type. In the ChlD phylogeny, the sequence of L. chlorophorum appeared to be excluded from the sequences of peridinin-containing dinoflagellates and those of the green algal/land plant sequences (Fig. 3A), suggesting that this sequence cannot be of VI-type or EAtype. Instead, the sequence of L. chlorophorum, as well as those of chromerids, cryptophytes and Eutreptiella, were placed within the radiation of the stramenopile sequences, and their monophyly was supported by a MLBP of $96 \%$ and a BPP of 0.99 . This tree topology prompts us to propose that L. chlorophorum ChlD was acquired from a stramenopile (i.e. LA-type).

To our surprise, the ChlH, POR and CS phylogenies placed the sequences of $L$. chlorophorum within the radiation of the chlorarachniophyte sequences, and their monophylies received MLBPs $>92 \%$ and by BPPs $>0.62$ (Figs. 3B, 3F and 3G). We here propose multiple gene transfers from chlorarachniophytes to the ancestral Lepidodinium cell to interpret the aforementioned tree topologies. The putative chlorarachniophyte origins of the three genes are not contradict to a recent molecular clock analysis, in which the green algal endosymbiosis leading to the Lepidodinium plastids was predicted to occur more recently than that leading to the chlorarachniophyte plastids (Jackson et al. 2018).

The F-DVR phylogeny reconstructed a robust affinity between the sequence of $L$. chlorophorum and the chlorarachniophyte clade within the land plant/green algal sequences (Fig. 3D). This tree topology requires a combination of an EGT and LGT, as Lepidodinium species and chlorarachniophytes acquired their plastids commonly from green algae. Either ancestral Lepidodinium cell or the ancestral chlorarachniophyte acquired an F-DVR gene from the green algal endosymbiont (i.e. EGT), followed by the second gene transfer between the two organisms (i.e. LGT). Thus, we have to leave the evolutionary origin of the L. chlorophorum F-DVR 
661

662

663

664

665

666

667

668

669

670

671

672

673

674

675

676

677

678

679

680

681

682

683

684

uncertain.

The phylogenetic analyses described above revealed that EGT was much less significant in the L. chlorophorum pathway than the kareniacean pathway (see above). Instead, chlorarachniophytes seemingly donated the genes encoding the proteins involved in four out of the five steps in the L. chlorophorum pathway.

\section{Non-mevalonate pathway for the IPP biosynthesis}

The origin of all the enzymes involved in the non-mevalonate pathway of Ke. brevis and $\mathrm{Kl}$. veneficum were investigated carefully in Bentlage et al. (2016). On the other hand, the entire picture of the L. chlorophorum pathway remain to be completed, leaving 5 out the 7 enzymes involved in this pathway unidentified (Minge et al. 2010). We successfully identified all the enzymes involved in the non-mevalonate pathway in L. chlorophorum (see below). In this section, we mainly examined the origins of individual enzymes involved in the non-mevalonate pathway of $L$. chlorophorum, coupled with a brief overview of the same pathway of the two kareniacean species. Thirty-one out of the 32 transcripts investigated here were found to possess N-terminal extensions, which likely work as plastid-localizing signals (Note that 12 sequences were proceeded by the putative SPs, and three of the non-SP portions were predicted to have the TP potential; see Table S5 for the details).

For the step synthesizing 1-deoxy-D-xylulose-5-phosphate (DXP) from pyruvate and glyceraldehyde 3-phosphate, L. chlorophorum was found to possess two versions of DXP synthase (DXS) (Lepidodinium-1 and 2). The DXS phylogeny robustly grouped the Lepidodinium-1 and 2 sequences with those of peridinin-containing dinoflagellates and Ke. brevis (Fig. 4A). The sequence of $\mathrm{Kl}$. veneficum was found to be remote from the dinoflagellate clade, and showed an 
685 affinity to the haptophyte sequences (Fig. 4A). The clade comprising the sequences of $K l$. 686 veneficum and haptophytes received a MLBP of $63 \%$ and a BPP of 0.82 (if the Pavlova sequence 687 was excluded, the "Karlodinium + haptophyte" clade was supported by a MLBP of 98\% and a BPP of 1.0). Thus, we conclude that the DXS sequences of L. chlorophorum and Ke. brevis were of VI-type, while that of $K l$. veneficum was of EA-type. DXP reductase (DXR). Minge et al. (2010) detected a partial sequence of a VI-type DXR (GenBank accession number CCC15090). From our transcriptome data, two versions of DXR were identified in L. chlorophorum (Lepidodinium-1 and 2; the former corresponds to the previously reported DXR sequence). The DXR phylogeny (Fig. 4B) reconstructed a robust monophyly of the Lepidodinium-1 and 2 sequences, the sequences of peridinin-containing dinoflagellates, and those of Ke. brevis and Kl. veneficum (MLBP of 100\% and BPP of 1.0), suggesting that L. chlorophorum and the two kareniacean species use VI-type proteins for this reaction. diphospho)-2-C-methyl-D-erythritol (CDP-ME). The Lepidodinium-1 sequence was concluded as LA-type based on its remote position from the sequences of peridinin-containing dinoflagellates or green plants in the IspD phylogeny (Fig. 4C). Instead, the Lepidodinium-1 sequence showed a specific affinity to the sequence of a stramenopile Ochromonas sp. with a MLBP of $100 \%$ and a BPP of 1.0. As the monophyly of the stramenopile sequences was not recovered probably due to insufficient phylogenetic signal in the IspD alignment, two scenarios remain possible. The simplest scenario assumes that the ispD gene was transferred between L. chlorophorum and Ochromonas sp. Alternatively, a scenario, in which invokes separate gene transfers from an as-yet-unknown organism to L. chlorophorum and Ochromonas sp., is also possible. To examine the two scenarios 
709 in the future, we need to prepare a new IspD alignment including the sequences from stramenopiles

710 closely related to Ochromonas sp. (i.e. chrysophycean algae) and reexamine a new alignment in

711 the future. In contrast, the Lepidodinium-2 sequence, together with a single sequence of $K l$. 712 veneficum, were considered as VI-type, as they formed a clade with those of peridinin-containing 713 dinoflagellates (MLBP of $81 \%$ and BPP of 0.68). The IspD sequence of Ke. Brevis appeared to be 714 nested within the clade of green algae/land plants and chlorarachniophytes, and being distantly 715 related to the dinoflagellate clade (including the sequences of L. chlorophorum and Kl. veneficum) 716 or the haptophyte clade (Fig. 4C). The position of Ke. brevis IspD is consistent with the green algal 717 origin of this enzyme proposed by Bentlage et al. (2016). Minge et al. (2010) reported a VI-type 4-diphosphocytidyl-2-C-methyl-D-erythritol 719 kinase (IspE) (GenBank accession number CCC15094), which phosphorylates CDP-ME to 2phospho-4-(cytidine 5'-diphospho)-2-C-methyl-D-erythritol (CDP-MEP), in L. chlorophorum. In this study, we detected two distinct versions of IspE (Lepidodinium-1 and 2) — the Lepidodinium1 sequence corresponds to the version reported in Minge et al. (2010) and the Lepidodinium-2 sequence is a novel version of IspE. The IspE phylogeny (Fig. 4D) recovered a monophyletic clade of the two sequences of $L$. chlorophorum, the sequences of peridinin-containing dinoflagellates, the sequence of $\mathrm{Kl}$. veneficum, and one of the two sequences of Ke. brevis (Karenia-2), which was supported by a MLBP of $88 \%$ and a BPP of 0.99 . Thus, L. chlorophorum, Ke. brevis and Kl. veneficum possess VI-type versions of IspE. In addition, Ke. brevis possesses an EA-type IspE 728 (Karenia-1), which was united with the haptophyte sequences with a MLBP of 100\% and a BPP 729 of 0.98 .

The ancestral dinoflagellate likely possessed two versions of 2-C-methyl-D-erythritol 2,4731 cyclodiphosphate synthase (IspF), which converts CDP-MEP to 2-C-methyl-D-erythritol 2,4732 cyclodiphosphate (MEcPP). In the IspF phylogeny (Fig. 4E), the vast majority of the 
733 dinoflagellates sequences was split into two clades (D1 and D2), of which monophylies were

734 supported by MLBPs of 85-94\% and BPPs of 0.99-1.0, respectively. D1 clade appeared to contain

735 one of the two sequences of L. chlorophorum (Lepidodinium-1), as well as the sequence of Kl.

736 veneficum and one of the two sequences of Ke. brevis (Karenia-2). The other version of $L$.

737 chlorophorum (Lepidodinium-2) was nested within D2 clade. Thus, we conclude that $L$.

738 chlorophorum, Ke. brevis and Kl. veneficum possess VI-type versions of IspF. As reported in

739 Bentlage et al. (2016), Ke. brevis possesses an additional IspF sequence (Karenia-1) with a

740 phylogenetic affinity to the haptophyte sequences, suggesting that this version is of EA-type.

741 The origin and evolution of 1-hydroxy-2-methyl-2-butenyl 4-diphosphate (HMB-PP)

742 synthase (IspG), which synthesize HMB-PP from MEcPP, seems straightforward in

743 dinoflagellates. We phylogenetically analyzed two versions of IspG in L. chlorophorum

744 (Lepidodinium-1 and 2) identified in this study, together with the sequences of Ke. brevis and Kl.

745 veneficum. In the IspG phylogeny (Fig. 4F), the aforementioned dinoflagellate sequences tightly

746 clustered with the sequences of peridinin-containing dinoflagellates (MLBP of 98\% and BPP of

747 1.0). Thus, we concluded that Ke. brevis, Kl. veneficum and L. chlorophorum uses VI-type

748 enzymes to synthesize MEcPP.

749 The last step of the non-mevalonate pathway is catalyzed by HMB-PP reductase (IspH)

750 to yield IPP from HMG-PP. We identified two versions of IspH in L. chlorophorum

751 (Lepidodinium-1 and 2). The IspH phylogeny (Fig. 4G) recovered a clade comprising the

752 sequences of peridinin-containing dinoflagellates, apicomplexan parasites and chromerids, as well

753 as the two versions of L. chlorophorum and one of the three versions of Ke. brevis (Karenia-3),

754 with a MLBP of $68 \%$ and a BPP of 0.92 . We noticed the rapidly evolving natures of the

755 apicomplexan and chromerid sequences, which highly likely biased the phylogenetic inference

756 from the IspH alignment. Consequently, the same alignment was reanalyzed after the exclusion of 
757 the long-branch sequences. In both ML and Bayesian analyses of the second IspH alignment, the

758 Lepidodinium-1 and 2, and Karenia-3 sequences grouped with those of peridinin-containing

759 dinoflagellates with a MLBP of $100 \%$ and a BPP of 0.99 (Fig. 4G). We conclude that the two

760 versions of IspH in L. chlorophorum are of VI-type. Ke. brevis was found to possess two additional

761 versions of IspH (Karenia-1 and Karenia-2). The IspH phylogeny (Fig. 4G) united the Karenia-1

762 sequence and a single IspH sequence of $K l$. veneficum with the sequences of haptophytes with a

763 MLBP of $90 \%$ and a BPP of 0.98 . The Karenia-2 sequence was excluded from the dinoflagellate

764 or haptophyte clade, and instead connected to the sequence of a stramenopile Ochromonas sp. with

765 a MLBP of $88 \%$ and a BPP of 0.99 (Fig. 4G). As the sequence of Ochromonas sp. showed no clear

766 affinity to other stramenopile sequences, the origin of the Karenia-2 sequence remains unclear.

767 Thus, as discussed in Bentlage et al. (2016), Ke. brevis uses VI-type (Karenia-3), EA-type

768 (Karenia-1) and LA-type (Karenia-2) enzymes to yield IPP, while Kl. veneficum possesses a 769 single, EA-type version.

770 As Bentlage et al. (2016) demonstrated, the non-mevalonate pathways in Ke. brevis and $771 \mathrm{Kl}$. veneficum are evolutionary hybrids of VI-type and EA-type enzymes. In sharp contrast, the 772 same pathway in L. chlorophorum appeared to be dominated by VI-type proteins, except a single 773 LA-type protein (one of the two IspD versions).

\section{Discussion}

Kl. veneficum pathways investigated here appeared to be composed of three evolutionary types of 780

\section{Perspectives toward the evolution of kareniacean dinoflagellates and their plastids}

As anticipated from the haptophyte origin of the kareniacean plastids, the Ke. brevis and proteins, namely EA-, VI- and LA-types (Patron et al. 2006; Nosenko et al. 2006; Hunsperger et 
781 al, 2015; Bentlage et al. 2016). Nevertheless, the impact of the genetic influx from the haptophyte 782 endosymbiont was different among the three pathways in Ke. brevis/Kl. veneficum (Fig. 5). In the two kareniacean species, EA-type proteins, together with a few LA-type proteins, found to dominate the $\mathrm{Chl} a$ biosynthesis, albeit no VI-type protein was detected. In sharp contrast, VI-type proteins persist in 5-6 out of the 7 steps required for the non-mevalonate pathway for the IPP biosynthesis, albeit the contributions of EA-type and LA-type proteins may not be negligible. The evolutionary chimerism is most advanced in heme biosynthesis, in which all the three protein types are identified (Fig. 5).

Based on the difference in degree of evolutionary chimerism among the three pathways discussed in the previous section, we here explore the early evolution of kareniacean species. Saldarriaga et al. (2001) hinted non-photosynthetic intermediate stages for the dinoflagellates bearing non-canonical plastids based on the dinoflagellate phylogeny inferred from small subunit ribosomal DNA sequences, albeit they made no mention of the presence/absence of a residual plastid. Patron et al. (2006) and Nosenko et al. (2006) hypothesized that the ancestral kareniacean species possessed a non-photosynthetic plastid prior to the haptophyte endosymbiosis, although both studies assessed a restricted number of plastid-localized proteins. The above proposal is plausible, as secondarily non-photosynthetic eukaryotes often possess plastids with no photosynthetic activity but diverse metabolic capacities including those to synthesize heme and/or IPP (Lim \& McFadden, 2010; Lohr et al, 2012; Kamikawa et al, 2015b, 2015c, 2017; Janouškovec et al, 2017). Notably, Kareniaceae includes a kleptoplastic species found in the Ross Sea, Antarctica, in addition to the species bearing haptophyte-derived plastids (Gast et al, 2006, 2007). The coexistence of the species bearing the haptophyte-derived non-canonical plastids and that leading a kleptoplastic lifestyle in the same family lends an additional support for the nonphotosynthetic nature of their common ancestor. The hypothesis for the non-photosynthetic nature 
805 in the ancestral kareniacean species can explain well the elimination of VI-type proteins from the 806 Chl a biosynthesis in both Ke. brevis and Kl. veneficum (Fig. 5). During the putative non807 photosynthetic period in the early kareniacean evolution, the proteins involved in the Chl $a$ 808 biosynthesis may have been dispensable, leading to discard of the corresponding genes from the 809 dinoflagellate genome. Interestingly, Patron et al. (2006) identified atpC transcript encoding a 810 subunit of VI-type ATP synthase in $\mathrm{Kl}$. veneficum, implying that ATP synthase on plastid 811 thylakoid membrane has not been discarded during tertiary endosymbiosis. Nevertheless, the 812 presence of VI-type ATP synthase in the current $\mathrm{Kl}$. veneficum plastid is not necessary to negate 813 the putative non-photosynthetic period for the early kareniacean evolution, as the plastid ATP 814 synthase can persist in secondarily non-photosynthetic organisms (Donaher et al, 2009; Wicke et al, 2013; Kamikawa et al, 2015c; 2017). An equivalent observation was also reported in a nonphotosynthetic cyanobacterium by Nakayama et al, (2014). In the later kareniacean evolution, the entire pathway for the Chl $a$ biosynthesis was most likely reconstructed in the haptophyte-derived plastid by incorporating exogenous genes (acquired mainly from the endosymbiont). In contrast, both Ke. brevis and Kl. veneficum seemingly use VI-type proteins to synthesize both heme and IPP, suggesting that the proteins originally worked in the peridinin-containing plastid persisted in the ancestral kareniacean species beyond the haptophyte endosymbiosis. The two pathways have been modified after the haptophyte endosymbiosis by incorporating exogenous genes acquired from phylogenetically diverse organisms (including the endosymbiont), as we observed both EAand LA-type proteins in the current pathways in both Ke. brevis and Kl. veneficum (Fig. 5). species possessed the residual genome as argued below. Poly-uridylation of the $3^{\prime}$ termini of plastid transcripts was found primarily in peridinin-containing dinoflagellates, but also Ke. brevis and $K l$. 
machinery for the RNA modification have been inherited from the photosynthetic ancestor bearing a peridinin-containing plastid to the extent kareniacean species beyond the putative nonphotosynthetic period. In line with the above speculation, the putative non-photosynthetic plastid in the ancestral kareniacean species most likely retained a transcriptionally active genome.

We can retrieve an additional insight into the early kareniacean evolution by comparing the phylogenetic inventories of VI-, EA- and LA-type proteins in the heme, Chl $a$ and IPP biosynthetic pathways between Ke. brevis and Kl. veneficum. For instance, the contribution of VItype proteins to the heme biosynthesis seemingly differs between Ke. brevis and Kl. veneficum, which retain 6 and 3 VI-type proteins for the 9 steps in the heme biosynthesis, respectively (Fig. 5). Coincidently, the significance of LA-type proteins in the particular pathway seems to be expanded in the Kl. veneficum pathway comparing to the Ke. brevis pathway. These observations suggest that the reconstruction of metabolic pathways in the haptophyte-derived plastids (i.e. gene acquisitions/losses) was not completed before the separation of the genera Karenia and Karlodinium. However, we need to assess carefully whether the differences between the Ke. brevis and $K l$. veneficum pathways observed in our comparisons stemmed from the incomplete coverages of gene repertories in the two kareniacean species in future studies. Alternatively, the differences between the Ke. brevis and $\mathrm{Kl}$. veneficum pathways observed in our comparisons might reflect the separate haptophyte endosymbioses in Karenia and Karlodinium, as a plastid small subunit ribosomal DNA (pl-SSU rDNA) phylogeny placed the two dinoflagellate species in two remote positions within the haptophyte clade (Gast et al, 2007). The paraphyly of Karenia and Karlodinium implied by the pl-SSU rDNA phylogeny is apparently contradict to phylogenetic analyses based on multiple plastid-encoded proteins, in which Ke. brevis and Kl. veneficum grouped together by excluding haptophytes (Gabrielsen et al, 2011; Dorrell and Howe 2012). The precise origin (or origins) of haptophyte-derived plastids needs to examine by plastid genome- 
853 based multigene alignments including broader members of Kareniaceae (e.g., Takayama spp.) and 854 haptophytes in the future.

855

\section{Perspectives toward the evolution of Lepidodinium and its plastids}

857

858

859

860

861

862

863

864

865

866

867

868

869

870

871

872

873

874

875

876

The phylogenetic inventories of VI-, EA- and LA-type proteins appeared to be different among the heme, Chl $a$ and IPP biosynthetic pathways in L. chlorophorum (Fig. 5). The IPP synthesis in this species retains VI-type proteins in all of the 7 steps, and no EA-type protein was found. In sharp contrast, the contribution of LA-type proteins to the Chl $a$ biosynthesis is likely much greater than that of VI- or EA-type proteins. The heme biosynthesis appeared to be distinct from the two pathways described above, as we detected both VI- and LA-type proteins but no EA-type protein. Interestingly, the trend, of which VI-type proteins contribute to the heme and IPP biosyntheses at much greater magnitudes than the Chl $a$ biosynthesis, is common between L. chlorophorum and Ke. brevis/Kl. veneficum (Fig. 5). Thus, as discussed the putative ancestral state of kareniacean species (see above), we speculate that the ancestral Lepidodinium, which engulfed a green algal endosymbiont, experienced a non-photosynthetic period and discarded most of the genes encoding proteins involved in the $\mathrm{Chl} a$ biosynthesis, but retained a non-photosynthetic plastid with the capacities for synthesizing both heme and IPP. Ferredoxin-NADP ${ }^{+}$reductase (FNR) involved in photosynthetic electron transport chain has been identified in photosynthetic, as well as secondarily non-photosynthetic organisms (Balconi et al, 2009; Nakayama et al, 2014). Thus, the transcript encoding plastid-type FNR identified in the L. chlorophorum transcriptome (Minge et al. 2010) does not contradict the putative non-photosynthetic period in the ancestral Lepidodinium species.

We unexpectedly revealed a potentially large contribution of chlorarachniophyte genes to the Chl a biosynthesis in L. chlorophorum (Fig. 5). In the organismal tree of eukaryotes, 
877 chlorarachniophytes and dinoflagellates belong to two distantly related taxonomic assemblages,

878 Rhizaria and Alveolata, respectively. Likewise, the current plastids in chlorarachniophytes and

879 Lepidodinium were derived from distinct green algal groups, ulvophytes and pedinophytes, 880 respectively (Suzuki et al, 2016; Kamikawa et al. 2015a). Thus, the relationship between their host 881 lineages or that between their endosymbiont lineages (plastids) can provide no ground for the 882 presence of chlorarachniophyte genes in the L. chlorophorum genome. If the ancestral Lepidodinium cell fed on chlorarachniophytes in the natural environment, such predator-prey relationship led to the genetic influx from the prey (chlorarachniophyte) genome to the predator (Lepidodinium) genome. Nevertheless, under the circumstance postulated above, gene transferred from chlorarachniophytes could not have been restricted to a single metabolic pathway. To understand the biological reasons for the genetic contribution from chlorarachniophytes to the Chl a biosynthesis in L. chlorophorum, we need to explore (i) potential interaction between dinoflagellates and chlorarachniophytes in the natural environment and (ii) the biochemical and/or physiological commonality in the proteins involved in the $\mathrm{Chl} a$ biosynthesis between chlorarachniophytes and L. chlorophorum. Nevertheless, our proposal for the presence of the gene transfers, needs to be re-assessed in the future. In particular, much broader sampling of ChlH, POR, CS and F-DVR sequences would be critical to infer the precise origins of the four "LA-type" proteins involved in the L. chlorophorum $\mathrm{Chl} a$ biosynthesis. Depending on the future $\mathrm{ChlH}$, POR, CS and F-DVR phylogenies with improved sequence sampling, we may need to revise their phylogenetic origins proposed in the current study.

We also noticed a clear difference in contribution of EA-type proteins to the three pathways between L. chlorophorum and Ke. brevis/Kl. veneficum (Fig. 5). EA-type proteins are most likely indispensable for the heme, Chl $a$ and IPP biosyntheses in Ke. brevis/Kl. veneficum. 
901 On the other hand, only MgPMT in the Chl $a$ biosynthetic pathway appeared to be of green algal

902 origin in L. chlorophorum. One potential factor, which could introduce the marked difference

903 between the L. chlorophorum and Ke. brevis/Kl. veneficum pathways, is the difference in plastid904 targeting signal of nucleus-encoded plastid-related proteins between their endosymbionts. In both 905 eukaryotes bearing primary plastids (e.g., green algae) and those bearing complex plastids (e.g., 906 haptophytes and dinoflagellates), the vast majority of plastid-related proteins are nucleus-encoded,

907

908

909

910

911

912

913 which are synthesized in the cytosol and localized to the plastid. In green algae, nucleus-encoded plastid-related proteins are synthesized with N-terminal extensions (i.e. transit peptides or TP), which act as the "tags" to pass through the two membranes surrounding their plastids (Patron \& Waller, 2007). On the other hand, the "tag" sequences, which enable nucleus-encoded proteins to localize in complex plastids surrounded by three or four membranes, are more complicated than green algal plastids (Bolte et al, 2009). Patron et al, (2005) revealed that dinoflagellates with peridinin-containing plastids and haptophytes appeared to share a bipartite structure of plastidtargeting signal, which is composed of signal peptide and the TP-like region. Consequently, without any substantial modification on plastid-targeting signals, the ancestral kareniacean species could have targeted the proteins encoded by endosymbiotically transferred genes back to the haptophyte-derived plastid. In contrast, nucleus-encoded plastid-related proteins in the green algal endosymbiont engulfed by the ancestral Lepidodinium unlikely possessed bipartite plastidtargeting signals. Thus, in the ancestral Lepidodinium, the proteins encoded by endosymbiotically transferred genes needed to acquire bipartite plastid-targeting signals to be localized in the green alga-derived plastid surrounded by four membranes. Altogether, we here propose the initial presence/absence of bipartite plastid-targeting signals was one of the major factors affecting the EGT in dinoflagellates bearing non-canonical plastids. As anticipated from the above scenario, many LA-type proteins acquired from diverse eukaryotes bearing complex plastids (e.g., 
925 stramenopiles and chlorarachniophytes) were identified in the heme, Chl $a$ and IPP biosynthetic

926 pathways in L. chlorophorum. Nevertheless, the factor discussed above may not be dominant

927 enough to exclude EA-type proteins from a green alga (e.g., MgPMT; Fig. 3C) and LA-type

928 proteins from bacteria (e.g., GSAT; Fig. 2B) from the plastid proteome in L. chlorophorum. In the

929 future, we need to expand the detailed phylogenetic analyses into the entire L. chlorophorum

930 plastid proteome to examine the hypothesis based on the biosynthetic pathways for heme, Chl $a$ 931 and IPP.

932

933 Conclusion

934

935 We here assessed the evolutionary origins of the proteins involved in the three plastid-localized pathways for the heme, Chl $a$ and IPP biosyntheses in two separate dinoflagellate lineages bearing

937

938

939

940

941

942

943

944

945

946

947

948 non-canonical plastids, namely kareniacean dinoflagellates bearing haptophyte-derived plastids (i.e. Ke. brevis and Kl. veneficum), and L. chlorophorum established a green alga-derived plastid. In each of the two dinoflagellate lineages, the three pathways have been modified differently during the process reducing an algal endosymbiont to a non-canonical plastid. We interpreted that the observed difference stemmed from the nature of the ancestral dinoflagellate engulfed a haptophyte/green algal endosymbiont. When individual pathways were compared between $L$. chlorophorum and Ke. brevis/Kl. veneficum, EGT appeared to contribute to the pathways in the former lineage much more substantially than those in the latter lineage. We proposed that this observation emerged partially from the structural difference in plastid-localizing signal (i.e. presence or absence of the SP) between the proteins acquired from the haptophyte endosymbiont and those from a green algal endosymbiont. The discussion based on the Ke. brevis and Kl. veneficum sequence data need to be reexamined in future studies incorporating the data from 
949 additional kareniacean species (e.g., members belonging to the genus Takayama), as well as their

950 relative operating kleptoplastidy (Gast et al, 2006, 2007).

951

952 Acknowledgements

953

954 We thank Dr. Euki Yazaki (University of Tsukuba, Japan) for helping the phylogenetic analyses 955 presented in this study. We also thank four reviewers for their constructive discussions and 956 suggestions on this work.

957

\section{References}

959 Balconi, E., Pennati, A., Crobu, D., Pandini, V., Cerutti, R., Zanetti, G. and Aliverti, A. 2009. The 960 ferredoxin-NADP+ reductase/ferredoxin electron transfer system of Plasmodium falciparum. 961 Federation of European Biochemical Societies Journal 276:3825-36. DOI: 10.1111/j.1742962 4658.2009.07100.x.

963

Beale, S.I. 1999. Enzymes of chlorophyll biosynthesis. Photosynthesis Research 60:43-73. DOI: https://doi.org/10.1023/A:1006297731456.

966

Bentlage, B., Rogers, T. S., Bachvaroff, T. R. and Delwiche, C. F. 2016. Complex ancestries of isoprenoid synthesis in dinoflagellates. Journal of Eukaryotic Microbiology 63:123-137. DOI:10.1111/jeu.12261.

Bjørnland, T., Haxo, F. T. and Liaaen-Jensen, S. 2003. Carotenoids of the Florida red tide dinoflagellate Karenia brevis. Biochemical Systematics and Ecology 31:1147-1162. DOI: 10.1016/S0305-1978(03)00044-9.

Bolte, K., Bullmann, L., Hempel, F., Bozarth, A., Zauner, S. and Maier, U. G. 2009. Protein targeting into secondary plastids. Journal of Eukaryotic Microbiology 56:9-15. DOI: 10.1111/j.1550-7408.2008.00370.x.

Burki, F., Imanian, B., Hehenberger, E., Hirakawa, Y., Maruyama, S. and Keeling, P. J. 2014. 
980 Endosymbiotic gene transfer in tertiary plastid-containing dinoflagellates. Eukaryotic Cell 13:246981 255. DOI: 10.1128/EC.00299-13.

982

Chen, G. E., Canniffe, D. P. and Hunter, C. N. 2017. Three classes of oxygen-dependent cyclase 984 involved in chlorophyll and bacteriochlorophyll biosynthesis. Proceedings of the National Academy of Sciences of the United States of America 114:6280-6285. DOI: 10.1073/pnas.1701687114.

987

Darriba, D., Taboada, G.L., Doallo, R. and Posada, D. 2011. ProtTest 3: fast selection of best-fit models of protein evolution. Bioinformatics 27:1164-1165. DOI: 10.1093/bioinformatics/btr088.

Donaher, N., Tanifuji, G., Onodera, N. T., Malfatti, S. A., Chain, P. S. G., Hara, Y. and Archibald, J. M. 2009. The complete plastid genome sequence of the secondarily nonphotosynthetic alga Cryptomonas paramecium: reduction, compaction, and accelerated evolutionary rate. Genome Biology and Evolution 1:439-448. DOI: 10.1093/gbe/evp047.

Dorrell, R. G., Gile, G., McCallum, G., Méheust, R., Bapteste, E. P., Klinger, C. M., Brilletand haptophytes revealed through an ancient plastid proteome. eLife 6:e23717. DOI: 10.7554/eLife.23717.

1004

1005

Dorrell, R. G., Hinksman, G. A. and Howe, C. J. 2016. Diversity of transcripts and transcript 1006 processing forms in plastids of the dinoflagellate alga Karenia mikimotoi. Plant Molecular Biology 90:233-247. DOI: 10.1007/s11103-015-0408-9.

1008

1009

Dorrell, R. G. and Howe, C. J. 2012. Functional remodeling of RNA processing in replacement chloroplasts by pathways retained from their predecessors. Proceedings of the National Academy of Sciences of the United States of America 109:18879-84. DOI: 10.1073/pnas.1212270109.

1012

Dubey, V. S., Bhalla, R. and Luthra, R. 2003. An overview of the non-mevalonate pathway for 1014 terpenoid biosynthesis in plants. Journal of Biosciences 28:637-646. 
1016 Emanuelsson, O., Nielsen, H., von Heijne, G. 1999. ChloroP, a neural network-based method for 1017 predicting chloroplast transit peptides and their cleavage sites. Protein Science: a Publication of 1018 the Protein Society 8:978-984. DOI: 10.1110/ps.8.5.978.

1019

1020 Fujita, Y. and Bauer, C. E. 2003. The light-independent protochlorophyllide reductase: a 1021 nitrogenase-like enzyme catalyzing a key reaction for greening in the dark. In: Kadish K. M., Smith 1022 K. M., Guilard, R. eds. The Porphyrin Handbook: Chlorophylls and bilins : biosynthesis, synthesis, 1023 and degradation. USA: Elsevier Science, 109-156.

1024

1025 Gabrielsen, T. M., Minge, M. A., Espelund, M., Tooming-Klunderud, A., Patil, V., Nederbragt, 1026 A. J., Otis, C., Turmel, M., Shalchian-Tabrizi, K., Lemieux, C. and Jakobsen, K. S. 2011. Genome 1027 evolution of a tertiary dinoflagellate plastid. PLoS ONE 6:e19132. DOI: 1028 10.1371/journal.pone.0019132.

1029

1030 Gast, R. J., Moran, D. M., Beaudoin, D. J., Dennett, M. R. and Caron, D. A. 2006. Abundance of 1031 a novel dinoflalgelate phylotype in the Ross Sea, Antarctica. Journal of Phycology, 42:233-242. 1032 DOI: $10.1111 / \mathrm{j} .1529 .8817 .2006 .00183 . x$.

1033

1034

1035

1036

1037

1038

1039

1040

1041

1042

1043

1044

1045

1046

1047

1048

1049

1050

1051
Gast, R. J., Moran, D. M., Dennett, M. R. and Caron, D. A. 2007. Kleptoplasty in an Antarctic dinoflagellate: caught in evolutionary transition? Environmental Microbiology, 9:39-45. DOI: 10.1111/j.1462-2920.2006.01109.x.

Haas, B. J., Papanicolaou, A., Yassour, M., Grabherr, M., Blood, P. D., Bowden, J., Couger, M. B., Eccles, D., Li, B., Lieber, M., Macmanes, M. D., Ott, M., Orvis, J., Pochet, N., Strozzi, F., Weeks, N., Westerman, R., William, T., Dewey, C. N., Henschel, R., Leduc, R. D., Friedman, N. and Regev, A. 2013. De novo transcript sequence reconstruction from RNA-seq using the Trinity platform for reference generation and analysis. Nature Protocols 8:1494-1512. DOI: 10.1038/nprot.2013.084.

Hoek, C., Mann, D. and Jahns, H. M. 1995. Algae: an introduction to phycology. Cambridge: Cambridge University Press.

Hunsperger, H. M., Randhawa, T. and Cattolico, R. A. 2015. Extensive horizontal gene transfer, duplication, and loss of chlorophyll synthesis genes in the algae. BMC Evolutionary Biology 15:16. DOI: $10.1186 / \mathrm{s} 12862-015-0286-4$. 
1052 Hunter, W. N. 2007. The non-mevalonate pathway of isoprenoid precursor biosynthesis.

1053 The Journal of Biological Chemistry 282:21573-21577. DOI: 10.1074/jbc.R700005200.

1054

1055

1056

1057

1058

1059

1060

1061

1062

1063

1064

1065

1066

1067

1068

1069

1070

1071

1072

1073

1074

1075

1076

1077

1078

1079

1080

1081

1082

1083

1084

1085

1086

1087

Ishida, K. and Green, B. R. 2002. Second- and third-hand chloroplasts in dinoflagellates: phylogeny of oxygen-evolving enhancer 1 (PsbO) protein reveals replacement of a nuclearencoded plastid gene by that of a haptophyte tertiary endosymbiont. Proceedings of the National Academy of Sciences of the United States of America 99:9294-9299. DOI: 10.1073/pnas.142091799.

Ito, H., Yokono, M., Tanaka, R. and Tanaka, A. 2008. Identification of a novel vinyl reductase gene essential for the biosynthesis of monovinyl chlorophyll in Synechocystis sp. PCC6803. The Journal of Biological Chemistry 283:9002-9011. DOI: 10.1074/jbc.M708369200.

Ito, H. and Tanaka A. 2014. Evolution of a new chlorophyll metabolic pathway driven by the dynamic changes in enzyme promiscuous activity. Plant and Cell Physiology 55:593-603. DOI: $10.1093 / \mathrm{pcp} / \mathrm{pct} 203$.

Jackson, C., Knoll, A. H., Chan, C. X., Verbruggen, H. 2018. Plastid phylogenomics with broad taxon sampling further elucidates the distinct evolutionary origins and timing of secondary green plastids. Scientific Reports 8:1523. DOI: 10.1038/s41598-017-18805-w.

Janouškovec, J., Horák, A., Oborník, M., Lukeš, J. and Keeling, P. J. 2010. A common red algal origin of the apicomplexan, dinoflagellate, and heterokont plastids. Proceedings of the National Academy of Sciences of the United States of America 107:10949-10954. DOI: 10.1073/pnas.1003335107.

Janouškovec, J., Gavelis, G. S., Burki, F., Dinh, D., Bachvaroff, T. R., Gornik, S. G., Bright, K. J., Imanian, B., Strom, S. L., Delwiche, C. F., Waller, R. F., Fensome, R. A., Leander, B. S., Rohwer, F. L. and Saldarriaga, J. F. 2017. Major transitions in dinoflagellate evolution unveiled by phylotranscriptomics. Proceedings of the National Academy of Sciences of the United States of America 114:E171-E180. DOI: 10.1073/pnas.1614842114.

Jeffrey, S. W., Sielicki, M. and Haxo, F. T. 1975. Chloroplast pigment patterns in dinoflagellates. Journal of Phycology 11: 374-384. DOI: 10.1111/j.1529-8817.1975.tb02799.x.

Kamikawa, R., Tanifuji, G., Kawachi, M., Miyashita, H., Hashimoto, T. and Inagaki, Y. 2015a. 
1088 Plastid genome-based phylogeny pinpointed the origin of the green-colored plastid in the 1089 dinoflagellate Lepidodinium chlorophorum. Genome Biology and Evolution 7:1133-1140. DOI: $1090 \quad 10.1093 / \mathrm{gbe} / \mathrm{evv060}$.

1091

Kamikawa, R., Yubuki, N., Yoshida, M., Taira, M., Nakamura, N., Ishida, K., Leander, B. S., 1093 Miyashita, H., Hashimoto, T., Mayama, S. and Inagaki, Y. 2015b. Multiple losses of 1094 photosynthesis in Nitzschia (Bacillariophyceae). Phycological Research 63:19-28. DOI: 1095 $10.1111 /$ pre. 12072 .

1096

Kamikawa R., Tanifuji G., Ishikawa S. A., Onodera N. T., Ishida K., Hashimoto T., Miyashita H., 1098 Mayama S. and Inagaki Y. 2015c. Proposal of a twin-arginine translocator system-mediated 1099 constraint against loss of ATP synthase genes from nonphotosynthetic plastid genomes. Molecular Biology and Evolution 32:2598-2604. DOI: 10.1093/molbev/msv134.

1101

Kamikawa R., Moog D., Zauner S., Tanifuji G., Ishida K., Miyashita H., Mayama S., Hashimoto 1103 T., Maier U. G., Archibald J. A. and Inagaki Y. 2017. A non-photosynthetic diatom reveals early 1104 steps of reductive evolution in plastids. Molecular Biology and Evolution 34:2355-2366. DOI: 10.1093/molbev/msx172.

Katoh, K. and Standley, D. M. 2013. MAFFT multiple sequence alignment software version 7: improvements in performance and usability. Molecular Biology and Evolution 30:772-780. DOI: 10.1093/molbev/mst010.

1110

Kobayashi, K., Masuda, T., Tajima, N., Wada, H. and Sato, N. 2014. Molecular phylogeny and 1112 intricate evolutionary history of the three isofunctional enzymes involved in the oxidation of protoporphyrinogen IX. Genome Biology and Evolution 6:2141-2155. DOI: 10.1093/gbe/evu170.

Kořený, L., Sobotka, R., Janouškovec, J., Keeling, P. J. and Oborník, M. 2011. Tetrapyrrole synthesis of photosynthetic chromerids is likely homologous to the unusual pathway of apicomplexan parasites. The Plant Cell 23:3454-3462. DOI: 10.1105/tpc.111.089102. the amino-acid replacement process. Molecular Biology and Evolution 21:1095-1109. DOI: 10.1093/molbev/msh112.

Lichtenthaler, H. K., Schwender, J., Disch, A. and Rohmer, M. 1997. Biosynthesis of isoprenoids 
1124 in higher plant chloroplasts proceeds via a mevalonate-independent pathway. Federation of 1125 European Biochemical Societies Letters 400:271-274. DOI: 10.1016/S0014-5793(96)01404-4.

1126

1127

1128

1129

1130

1131

1132

1133

1134

1135

1136

1137

1138

1139

1140

1141

1142

1143

1144

1145

1146

1147

1148

1149

1150

1151

1152

1153

1154

1155

1156

1157

1158

1159
Lim, L. and McFadden, G. I. 2010. The evolution, metabolism and functions of the apicoplast. Philosophical Transactions of the Royal Society of London. Series B, Biological Sciences 365:749-763. DOI: 10.1098/rstb.2009.0273.

Lohr, M., Im, C. S. and Grossman, A. R. 2005. Genome-based examination of chlorophyll and carotenoid biosynthesis in Chlamydomonas reinhardtii. Plant Physiology 138:490-515. DOI: 10.1104/pp.104.056069.

Lohr, M., Schwender, J. and Polle, J. E. 2012. Isoprenoid biosynthesis in eukaryotic phototrophs: a spotlight on algae. Plant Science 185-186:9-22. DOI: 10.1016/j.plantsci.2011.07.018.

Marron, A. O., Ratcliffe, S., Wheeler, G. L., Goldstein, R. E., King. N., Not, F., de Vargas, C. and Richter, D. J. 2016. The Evolution of Silicon Transport in Eukaryotes. Molecular Biology and Evolution 33:3226-3248. DOI: 10.1093/molbev/msw209.

Matsumoto, T., Shinozaki, F., Chikuni, T., Yabuki, A., Takishita, K., Kawachi, M., Nakayama, T., Inouye, I., Hashimoto, T. and Inagaki Y. 2011. Green-colored plastids in the dinoflagellate genus Lepidodinium are of core chlorophyte origin. Protist 162:268-276. DOI: 10.1016/j.protis.2010.07.001.

Meguro, M., Ito, H., Takabayashi, A., Tanaka, R. and Tanaka, A. 2011. Identification of the 7hydroxymethyl chlorophyll a reductase of the chlorophyll cycle in Arabidopsis. The Plant Cell 23:3442-3453. DOI: 10.1105/tpc.111.089714.

Minge, M. A., Shalchian-Tabrizi, K., Tørresen, O. K., Takishita, K., Probert, I., Inagaki, Y., Klaveness, D. and Jakobsen K. S. 2010. A phylogenetic mosaic plastid proteome and unusual plastid-targeting signals in the green-colored dinoflagellate Lepidodinium chlorophorum. BMC Evolutionary Biology 10:191. DOI: 10.1186/1471-2148-10-191.

Nagata, N., Tanaka, R., Satoh, S. and Tanaka, A. 2005. Identification of a vinyl reductase gene for chlorophyll synthesis in Arabidopsis thaliana and implications for the evolution of Prochlorococcus species. The Plant Cell 17:233-240. DOI: 10.1105/tpc.104.027276. 
1160 Nakayama, T., Kamikawa, R., Tanifuji G., Kashiyama, Y., Ohkouchi, N., Archibald, J. M. and 1161 Inagaki, Y. 2014. Complete genome of a nonphotosynthetic cyanobacterium in a diatom reveals 1162 recent adaptations to an intracellular lifestyle. Proceeding of the National Academy of Science of 1163 the United States of America 111:11407-11412. DOI: 10.1073/pnas.1405222111.

1164

1165 Nosenko, T., Lidie, K. L., Van Dolah, F. M., Lindquist, E., Cheng, J. F. and Bhattacharya, D. 2006. 1166 Chimeric plastid proteome in the Florida "red tide" dinoflagellate Karenia brevis. Molecular 1167 Biology and Evolution, 23: 2026-2038. DOI: 10.1093/molbev/ms1074.

Nymark, M., Valle, K. C., Brembu, T., Hancke, K., Winge, P., Andresen, K., Johnsen, G. and Bones, A. M. 2009. An integrated analysis of molecular acclimation to high light in the marine diatom Phaeodactylum tricornutum. PLoS ONE 4:e7743. DOI: 10.1371/journal.pone.0007743.

Oborník, M. and Green, B. R. 2005. Mosaic origin of the heme biosynthesis pathway in photosynthetic eukaryotes. Molecular Biology and Evolution 22:2343-2353. DOI: 10.1093/molbev/msi230.

1176

1177

Panek, H. and O'Brian, M. R. 2002. A whole genome view of prokaryotic haem biosynthesis. Microbiology 148:2273-2282. DOI: 10.1099/00221287-148-8-2273.

Patron, N. J, Waller, R. F., Archibald, J. M. and Keeling, P. J. 2005. Complex protein targeting to dinoflagellate plastids. Journal of Molecular Biology 348:1015-1024. DOI: 10.1016/j.jmb.2005.03.030.

1183

Patron, N. J., Waller, R. F. and Keeling, P. J. 2006. A tertiary plastid uses genes from two endosymbionts. Journal of Molecular Biology 357:1373-1382. DOI: 10.1016/j.jmb.2006.01.084.

Patron, N. J and Waller, R. F. 2007. Transit peptide diversity and divergence: A global analysis of plastid targeting signals. BioEssays 29:1048-58. DOI: 10.1002/bies.20638.

Petersen, T. N., Brunak, S., von Heijne, G. and Nielsen, H. 2011. SignalP 4.0: Discriminating 1191 signal peptides from transmembrane regions. Nature Methods 8:785-786. DOI: $119210.1038 /$ nmeth.1701. biosynthesis. European Journal of Biochemistry 237:323-343. DOI: 10.1111/j.1432- 
1196

1197

1198

1199

1200

1201

1202

1203

1204

1205

1206

1207

1208

1209

1210

1211

1212

1213

1214

1215

1216

1217

1218

1219

1220

1221

1222

1223

1224

1225

1226

1227

1228

1229

1230

1231

1033.1996.00323.x.

Richardson, E., Dorrell, R. G. and Howe, C. J. 2014. Genome-wide transcript profiling reveals the coevolution of plastid gene sequences and transcript processing pathways in the fucoxanthin dinoflagellate Karlodinium veneficum. Molecular Biology and Evolution 31:2376-2386. DOI: 10.1093/molbev/msu189.

Saldarriaga, J. F., Taylor, F. J., Keeling, P. J. and Cavalier-Smith, T. 2001. Dinoflagellate nuclear SSU rRNA phylogeny suggests multiple plastid losses and replacements. Journal of Molecular Evolution 53:204-213. DOI: 10.1007/s002390010210.

Sousa, F. L., Shavit-Grievink, L., Allen, J. F. and Martin, W. F. 2013. Chlorophyll biosynthesis gene evolution indicates photosystem gene duplication, not photosystem merger, at the origin of oxygenic photosynthesis. Genome Biology and Evolution 5:200-216. DOI: 10.1093/gbe/evs127.

Stamatakis, A. 2014. RAxML version 8: a tool for phylogenetic analysis and post-analysis of large phylogenies. Bioinformatics 30:1312-1313. DOI: 10.1093/bioinformatics/btu033.

Suzuki, S., Hirakawa, Y., Kofuji, R., Sugita, M. and Ishida, K. 2016. Plastid genome sequences of Gymnochlora stellata, Lotharella vacuolata, and Partenskyella glossopodia reveal remarkable structural conservation among chlorarachniophyte species. Journal of Plant Research 129:581590. DOI: 10.1007/s10265-016-0804-5.

Takishita, K., Ishida, K. and Maruyama, T. 2004. Phylogeny of nuclear-encoded plastid-targeted gapdh gene supports separate origins for the peridinin- and the fucoxanthin derivative-containing plastids of dinoflagellates. Protist 155:447-458. DOI: 10.1078/1434461042650325.

Takishita, K., Kawachi, M., Noël, M. H., Matsumoto, T., Kakizoe, N., Watanabe, M. M., Inouye, I., Ishida, K., Hashimoto, T. and Inagaki, Y. 2008. Origins of plastids and glyceraldehyde-3phosphate dehydrogenase genes in the green-colored dinoflagellate Lepidodinium chlorophorum. Gene 410:26-36. DOI: 10.1016/j.gene.2007.11.008.

Taylor, F. J. R., Hoppenrath, M. and Saldarriaga, J. F. 2008. Dinoflagellate diversity and distribution. Biodiversity and Conservation 17:407-418. DOI: 10.1007/s10531-007-9258-3.

Tengs, T., Dahlberg, O. J., Shalchian-Tabrizi, K., Klaveness, D., Rudi, K., Delwiche, C. F. and 
1232 Jakobsen, K. S. 2000. Phylogenetic analyses indicate that the 19' hexanoyloxy-fucoxanthin1233 containing dinoflagellates have tertiary plastids of haptophyte origin. Molecular Biology and 1234 Evolution 17:718-729. DOI: 10.1093/oxfordjournals.molbev.a026350.

1235

1236

Watanabe, M. M., Takeda, Y., Sasa, T., Inouye, I., Suda, S., Sawaguchi, T. and Chihara, M. 1987.

1237 A green dinoflagellate with chlorophylls $a$ and $b$ : morphology, fine structure of the chloroplast and

1238 chlorophyll composition. Journal of Phycology,23(s2):382-389. DOI: 10.1111/j.15291239 8817.1987.tb04148.x.

1240

1241

Watanabe, M. M., Suda, S., Inouye, I., Sawaguchi, T. and Chihara, M. 1990. Lepidodinium viride 1242 gen. et sp. nov. (Gymnodinaiales, Dinophyta), a green dinoflagellate with a chlorophyll $a$ - and $b$ -

1243

1244

1245

1246

1247

1248

1249

1250

1251

1252

1253

1254

1255

1256

1257

1258

1259

1260

1261

1262

1263

1264

1265

1266

1267

containing endosymbiont. Journal of Phycology 26:741-751. DOI: 10.1111/j.00223646.1990.00741.x.

Waterhouse, R. M., Seppey, M., Simão, F. A., Manni, M., Ioannidis, P., Klioutchnikov, G., Kriventseva, E. V. and Zdobnov, E. M. 2017. BUSCO applications from quality assessments to gene prediction and phylogenomics. Molecular Biology and Evolution 35:543-548. DOI: 10.1093/molbev/msx319.

Wicke, S., M€uller, K. F., de Pamphilis, C. W., Quandt, D., Wickett, N. J., Zhang, Y., Renner, S. S. and Schneeweiss, G. M. 2013. Mechanisms of functional and physical genome reduction in photosynthetic and nonphotosynthetic parasitic plants of the Broomrape family. Plant Cell 25:3711-3725. DOI: 10.1105/tpc.113.113373.

Wilhelm, C., Büchel, C., Fisahn, J., Goss, R., Jakob, T., Laroche, J., Lavaud, J., Lohr, M., Riebesell, U., Stehfest, K., Valentin, K. and Kroth, P. G. 2006. The regulation of carbon and nutrient assimilation in diatoms is significantly different from green algae. Protist 157:91-124. DOI: $10.1016 /$ j.protis.2006.02.003.

Yamanashi, K., Minamizaki, K. and Fujita, Y. 2015. Identification of the chlE gene encoding oxygen-independent Mg-protoporphyrin IX monomethyl ester cyclase in cyanobacteria. Biochemical and Biophysical Research Communications 463:1328-1333. DOI: 10.1016/j.bbrc.2015.06.124.

Zapata, M., Fraga, S., Rodríguez, F. and Garrido, J. L. 2012. Pigment-based chloroplast types in dinoflagellates. Marine Ecology Progress Series 465:33-52. DOI: 10.3354/meps09879. 


\section{Legends for Figures}

1270

1271 Fig 1. Enzymes examined in this study, and their substrates and products.

1272 C5 pathway for the heme biosynthesis is shaded in yellow. In this study, we regard the steps converting protoporphyrin IX to $\mathrm{Chl} a$ as the "Chl $a$ biosynthetic pathway," and shaded in green. The non-mevalonate pathway is shaded in blue. The first two pathways belong to "Porphyrin and chlorophyll metabolism" (map00860), while the third pathway belongs to "Terpenoid backbone biosynthesis" (map00900) in Kyoto Encyclopedia of Genes and Genomes pathway (KEGG pathway, http://www.genome.jp/kegg/pathway.html). Enzymes involved in the three pathways, as well as their gene names and corresponding KOIDs, are shown in red.

Fig 2. Maximum-likelihood phylogenies of 9 proteins involved in C5 pathway for the heme 1281 biosynthesis.

1282 We provide the maximum-likelihood bootstrap values (MLBPs), as well as Bayesian posterior probabilities (BPPs), only for the selected nodes, which are important to infer the origins of the proteins of Karenia brevis, Karlodinium veneficum and Lepidodinium chlorophorum. Dash marks represent the corresponding BPPs $<0.50$. MLBPs and BPPs are shown above and beneath the corresponding nodes, respectively. Subtrees/branches are color-coded (see the inset for the details). Statistically supported clades of the dinoflagellate, haptophyte and green plant sequences are highlighted by red, yellow and green backgrounds, respectively. Clades comprising the sequences of heterotrophic eukaryotes, which were predicted to be involved in C4 pathway, were shaded in gray. A. glutamyl-tRNA reductase (GTR).

B. glutamate-1-semialdehyde 2,1- 
1292 deaminase (PBGD). E. uroporphyrinogen III synthase (UROS). F. uroporphyrinogen

1293 decarboxylase (UROD). G. coproporphyrinogen oxidase (CPOX). H. protoporphyrinogen IX 1294 oxidase (PPOX) and I. ferrochelatase $(\mathrm{FeCH})$. The identical ML trees with full sequence names 1295 and MLBPs $\geq 50 \%$ are provided as the supplementary materials. Note that the substitution rates of 1296 the dashed branches are different from that of the solid lines as indicated in each figure.

1298 Fig 3. Maximum-likelihood phylogenies of 7 proteins involved in the Chl $a$ biosynthetic pathway. 1299 The details of this figure are same as those of Fig. 2. A. ChlD, one of the two nucleus-encoded 1300 subunits of $\mathrm{Mg}$-chelatase $(\mathrm{MgCH})$. B. $\mathrm{ChlH}$, the other nucleus-encoded subunit of $\mathrm{MgCH}$. C. $S$ -

1301

1302

1303

1304

1305

1306

1307

1308

1309

1310

1311

1312 for IPP biosynthesis.

1313 The details of this figure are same as those of Fig. 2. A. 1-deoxy-D-xylulose-5-phosphate (DXP)

1314 synthase (DXS). B. DXP reductoisomerase (DXR). C. 2-C-methyl-D-erythritol 4-phosphate 1315 cytidylyltransferase (IspD). D. 4-diphosphocytidyl-2-C-methyl-D-erythritol kinase (IspE). E. 2- 
1316 C-methyl-D-erythritol 2,4-cyclodiphosphate synthase (IspF). F. 1-hydroxy-2-methyl-2-butenyl 4-

1317 diphosphate (HMB-PP) synthase (IspG). G. HMB-PP reductase (IspH). The identical ML trees

1318 with full sequence names and MLBPs $\geq 50 \%$ are provided as the supplementary materials. Note 1319 that the substitution rates of the dashed branches are different from that of the solid lines as 1320 indicated in each figure.

1322 Fig 5. Overview of the origins of proteins involved in three plastid-localized biosynthetic pathways 1323 in Karenia brevis, Karlodinium veneficum and Lepidodinium chlorophorum.

1324 The origins of proteins of interest were classified into three types, (i) "VI-type" which were 1325 vertically inherited from the ancestral dinoflagellate beyond haptophyte/green algal 1326 endosymbiosis, (ii) "EA-type" which were acquired from the endosymbiont, and (iii) "LA-type" 1327 which were acquired from organisms distantly related to the host (dinoflagellates) or 1328 endosymbiont (haptophytes or green algae). Squares indicate the numbers and types of proteins of 1329 interest in the three dinoflagellates. In case of multiple versions being identified in one species, 1330 the numbers of the versions are shown in the corresponding squares. The squares are color-coded 1331 as follows: red, VI-type protein; yellow, EA-type in Karenia brevis and Karlodinium veneficum; 1332 green, EA-type in Lepidodinium; grey, LA-type. For DVR involved in the Chl $a$ biosynthetic 1333 pathway, we distinguish N-DVR and F-DVR by labeling " $\mathrm{N}$ " and "F," respectively. The open 1334 squares in the fourth lows labeled with question marks represent the sequences of which origins 1335 remain uncertain. 


\section{Figure 1 (on next page)}

Enzymes examined in this study, and their substrates and products.

C5 pathway for the heme biosynthesis is shaded in yellow. In this study, we regard the steps converting protoporphyrin IX to $\mathrm{Chl}$ a as the "Chl a biosynthetic pathway," and shaded in green. The non-mevalonate pathway is shaded in blue. The first two pathways belong to "Porphyrin and chlorophyll metabolism" (map00860), while the third pathway belongs to "Terpenoid backbone biosynthesis" (map00900) in Kyoto Encyclopedia of Genes and Genomes pathway (KEGG pathway, http://www.genome.jp/kegg/pathway.html). Enzymes involved in the three pathways, as well as their gene names and corresponding KOIDs, are shown in red. 


\section{Porphyrin and chlorophyll metabolism} (map00869)

\section{Heme biosynthesis}

\section{Glu-tRNA}

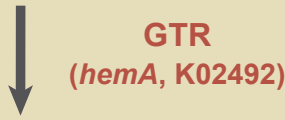

Glutamate-1-semialdehyde

$$
\begin{gathered}
\text { GSAT } \\
\text { (hemL, K01845) }
\end{gathered}
$$

5-Aminolevulinate

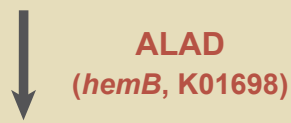

Porphobilinogen

$$
\begin{gathered}
\text { PBGD } \\
\text { (hem C, K01749) }
\end{gathered}
$$

Hydroxymethyl-

bilane

$$
\begin{gathered}
\text { UROS } \\
\text { (hemD, K01719) }
\end{gathered}
$$

Uroporphyrin III

$$
\begin{gathered}
\text { UROD } \\
\text { (hemE, K01599) }
\end{gathered}
$$

Coproporphyrin III

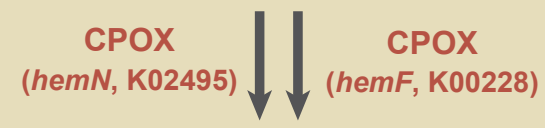

Protoporphyrinogen IX

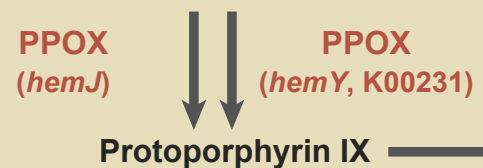

Protoporphyrin IX

\section{$\mathrm{FeCH}$}

(hemH, K01772)

\section{Chl a biosynthesis}

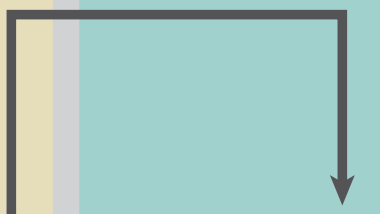

$\mathrm{MgCH}$

(chID, K03404/

chIH, K03403/

chll, K03405)

Mg-protoporphyrin IX

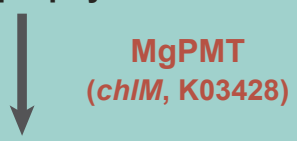

Mg-protoporphyrin IX monomethyl ester

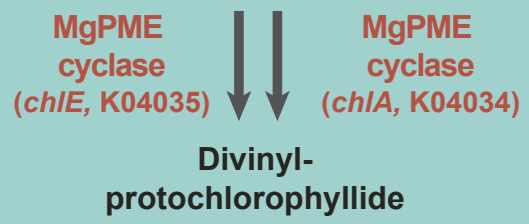

Divinyl-

protochlorophyllide

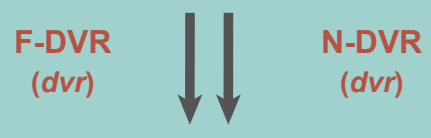

Protochlorophyllide

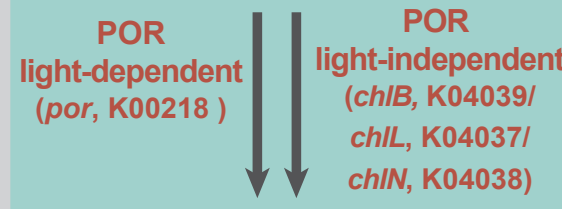

Chlorophyllide a

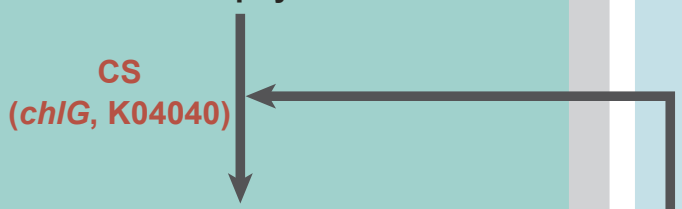

ChI a
Terpenoid backbone

biosynthesis

(map00900)

\section{IPP biosynthesis}

GAPDH + pyruvate

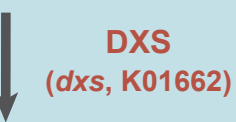

1-Deoxy-D-xylulose

5-phosphate

$$
\begin{gathered}
\text { DXR } \\
\text { (isp C, K00099) }
\end{gathered}
$$

2-C-Methyl-D-erythritol

4-phosphate

$$
\begin{gathered}
\text { IspD } \\
\text { (ispD, K00991) }
\end{gathered}
$$

4-(Cytidine 5'-diphospho)-

2-C-methyl-D-erythritol

$$
\downarrow\left(\begin{array}{c}
\text { IspE } \\
\text { (ispE, K00919) }
\end{array}\right.
$$

2-Phospho-4-(cytidine 5'-diphospho)-

2-C-methyl-D-erythritol

$$
\begin{gathered}
\text { IspF } \\
(\text { ispF, K01770) }
\end{gathered}
$$

\section{2-C-Methyl-D-erythritol}

2,4-cyclodiphosphate

$$
\begin{gathered}
\text { IspG } \\
\text { (ispG, K03526) }
\end{gathered}
$$

1-Hydroxy-2-methyl2-butenyl 4-diphosphate

$$
\checkmark(\text { ispH, K03527) }
$$

\section{Protoheme}




\section{Figure 2 (on next page)}

Maximum-likelihood phylogenies of 9 proteins involved in C5 pathway for the heme biosynthesis.

We provide the maximum-likelihood bootstrap values (MLBPs), as well as Bayesian posterior probabilities (BPPs), only for the selected nodes, which are important to infer the origins of the proteins of Karenia brevis, Karlodinium veneficum and Lepidodinium chlorophorum. Dash marks represent the corresponding BPPs $<0.50$. MLBPs and BPPs are shown above and beneath the corresponding nodes, respectively. Subtrees/branches are color-coded (see the inset for the details). Statistically supported clades of the dinoflagellate, haptophyte and green plant sequences are highlighted by red, yellow and green backgrounds, respectively. Clades comprising the sequences of heterotrophic eukaryotes, which were predicted to be involved in C4 pathway, were shaded in gray. A. glutamyl-tRNA reductase (GTR). B. glutamate-1-semialdehyde 2,1-aminomutase (GSAT). C. delta-aminolevulinic acid dehydratase (ALAD). D. porphobilinogen deaminase (PBGD). E. uroporphyrinogen III synthase (UROS). F. uroporphyrinogen decarboxylase (UROD). G. coproporphyrinogen oxidase (CPOX). H. protoporphyrinogen IX oxidase (PPOX) and I. ferrochelatase (FeCH). The identical ML trees with full sequence names and MLBPs $\geq 50 \%$ are provided as the supplementary materials. Note that the substitution rates of the dashed branches are different from that of the solid lines as indicated in each figure. 
ABorerJ
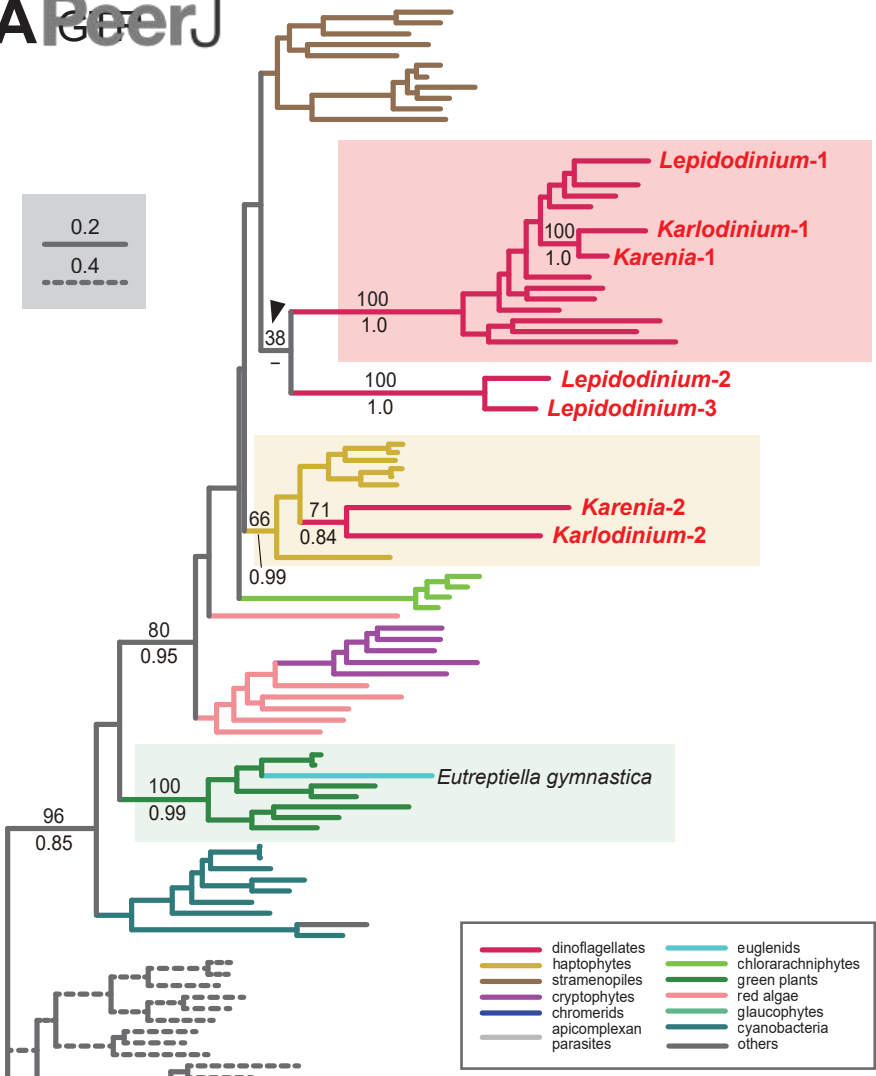
$r=0=0$
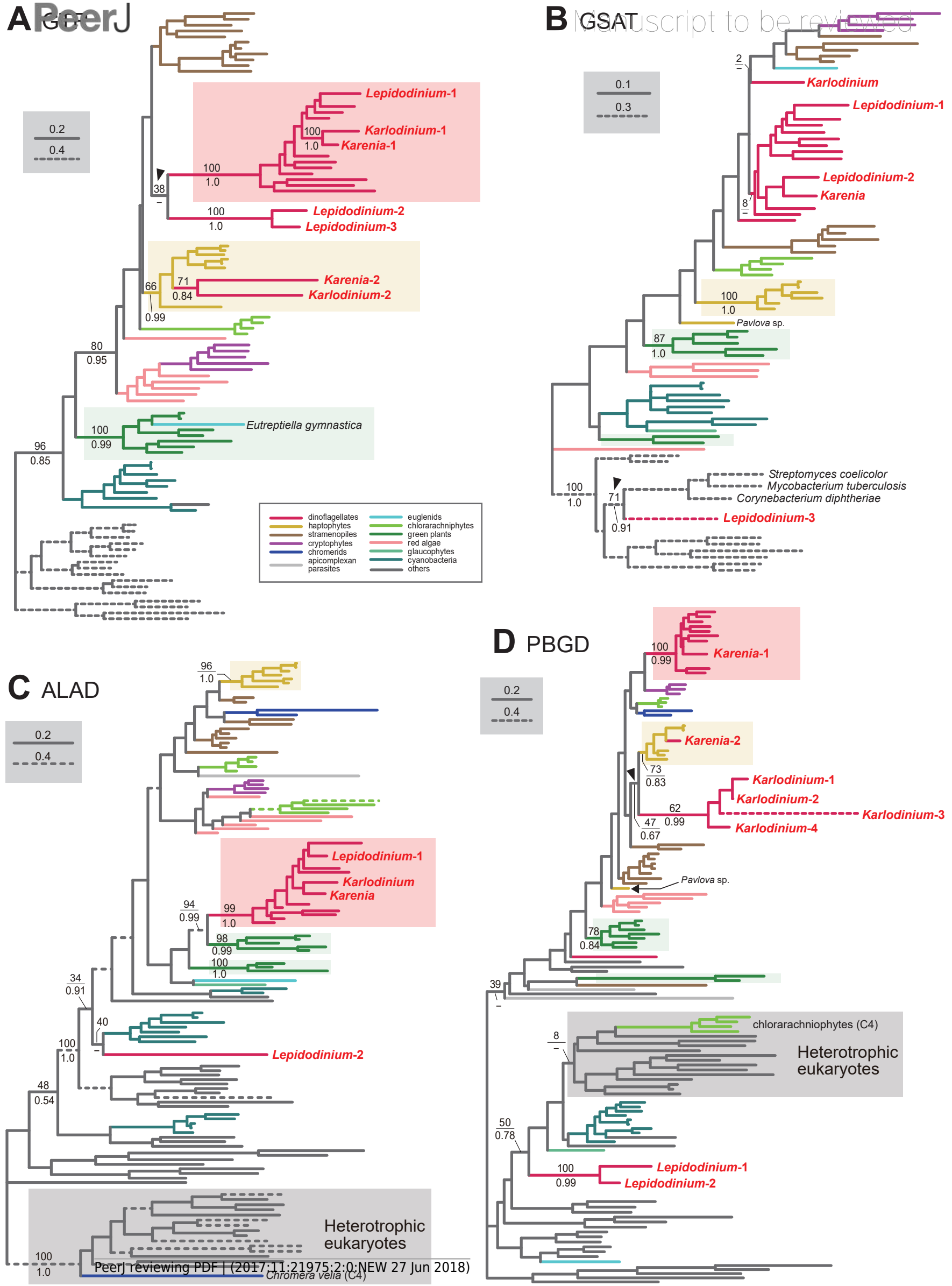
E

UROS

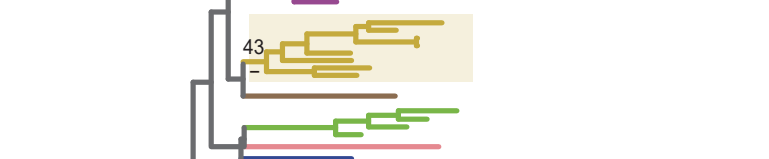

\section{$\mathbf{G} \operatorname{cPOX}$}
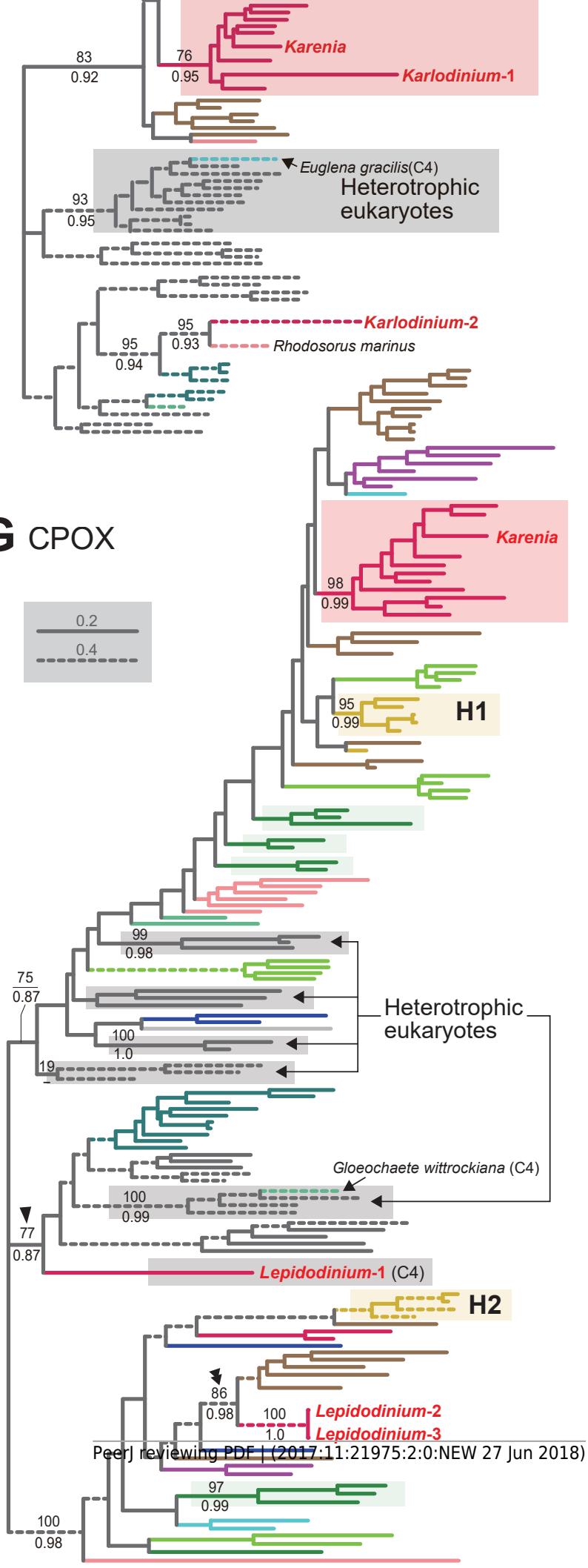

Karlodinium-2

Heterotrophic eukaryotes

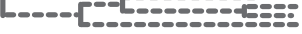

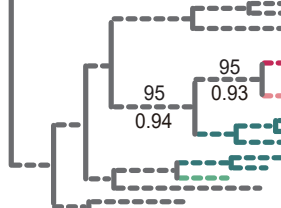

-

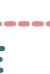

- Rhodos
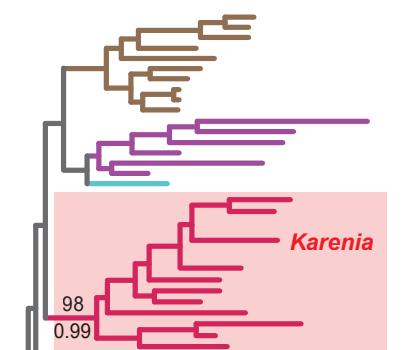

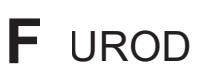

$100 \cdot 1.0$

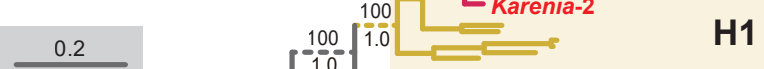

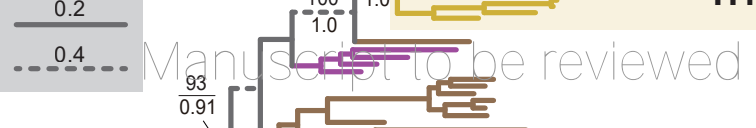

$78 \frac{99-2=0}{0.99-2}$
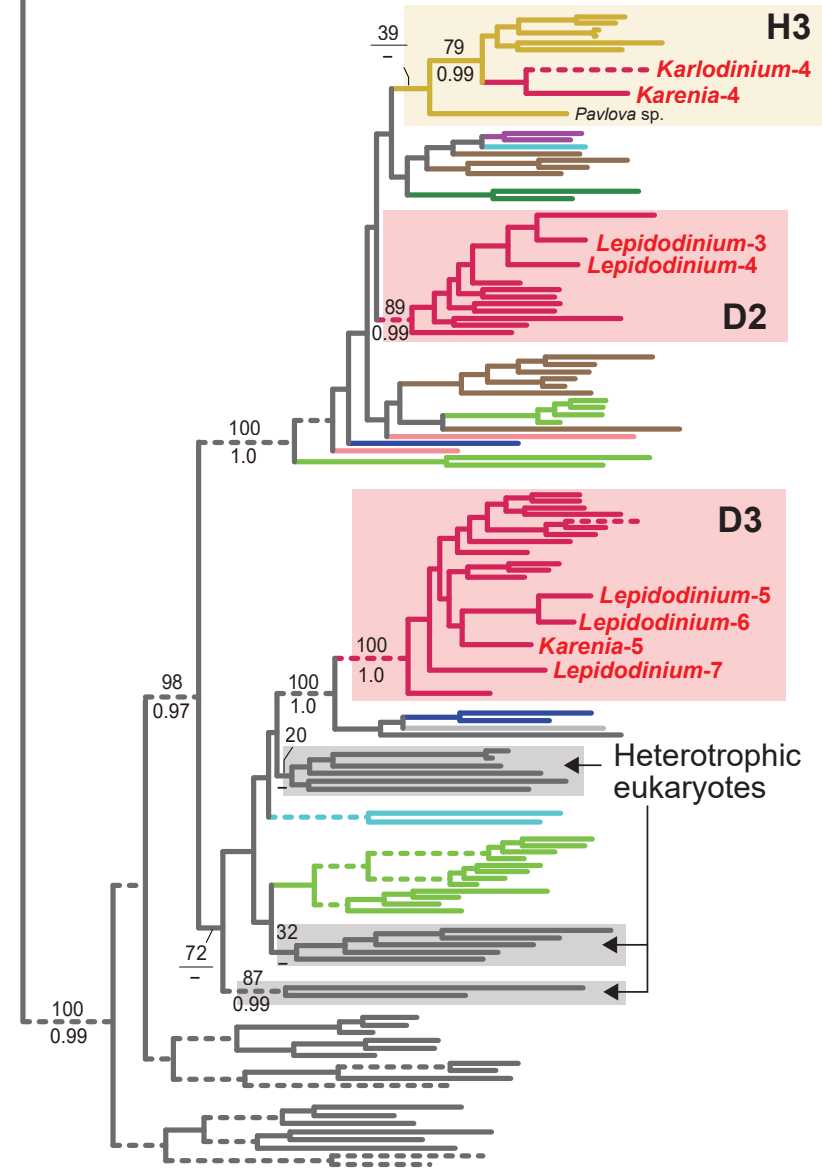


\section{PeerJ}

H PPOX

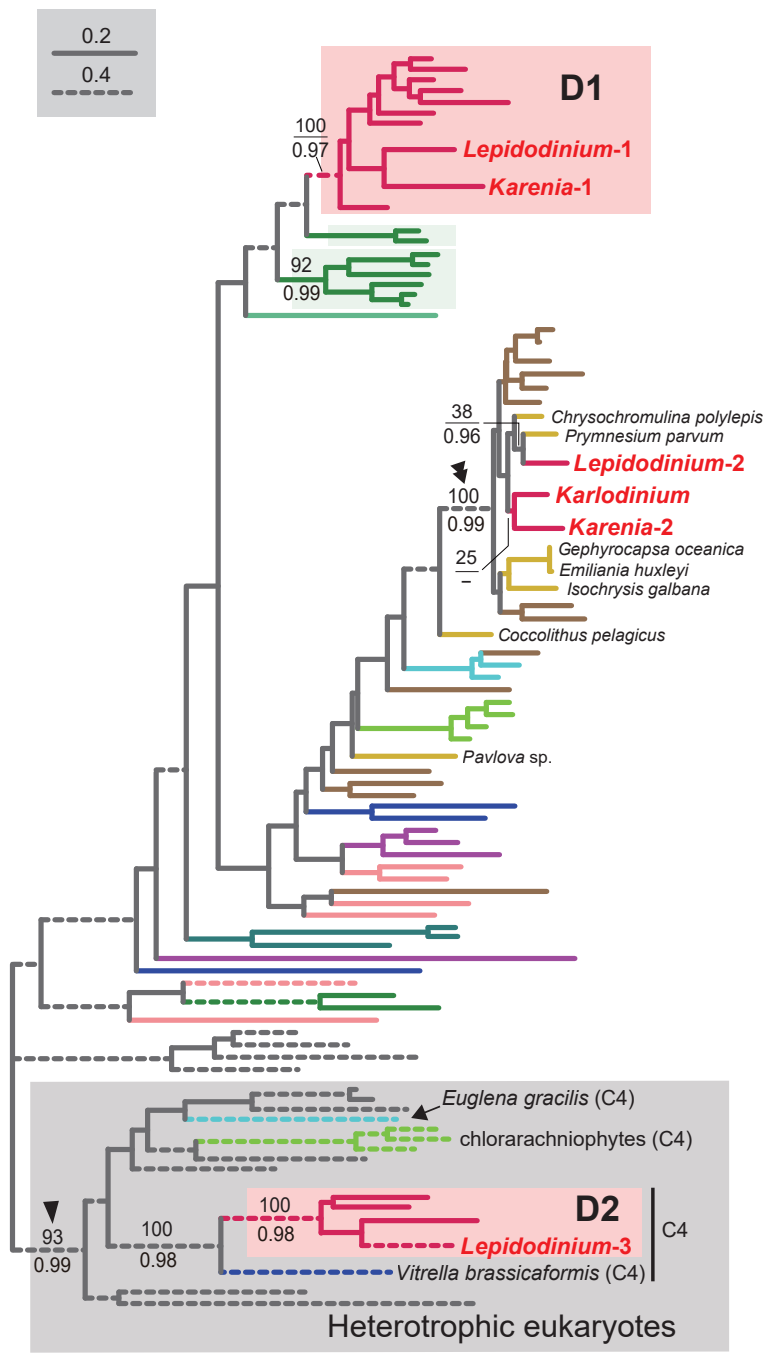

Manuscript to be reviewed
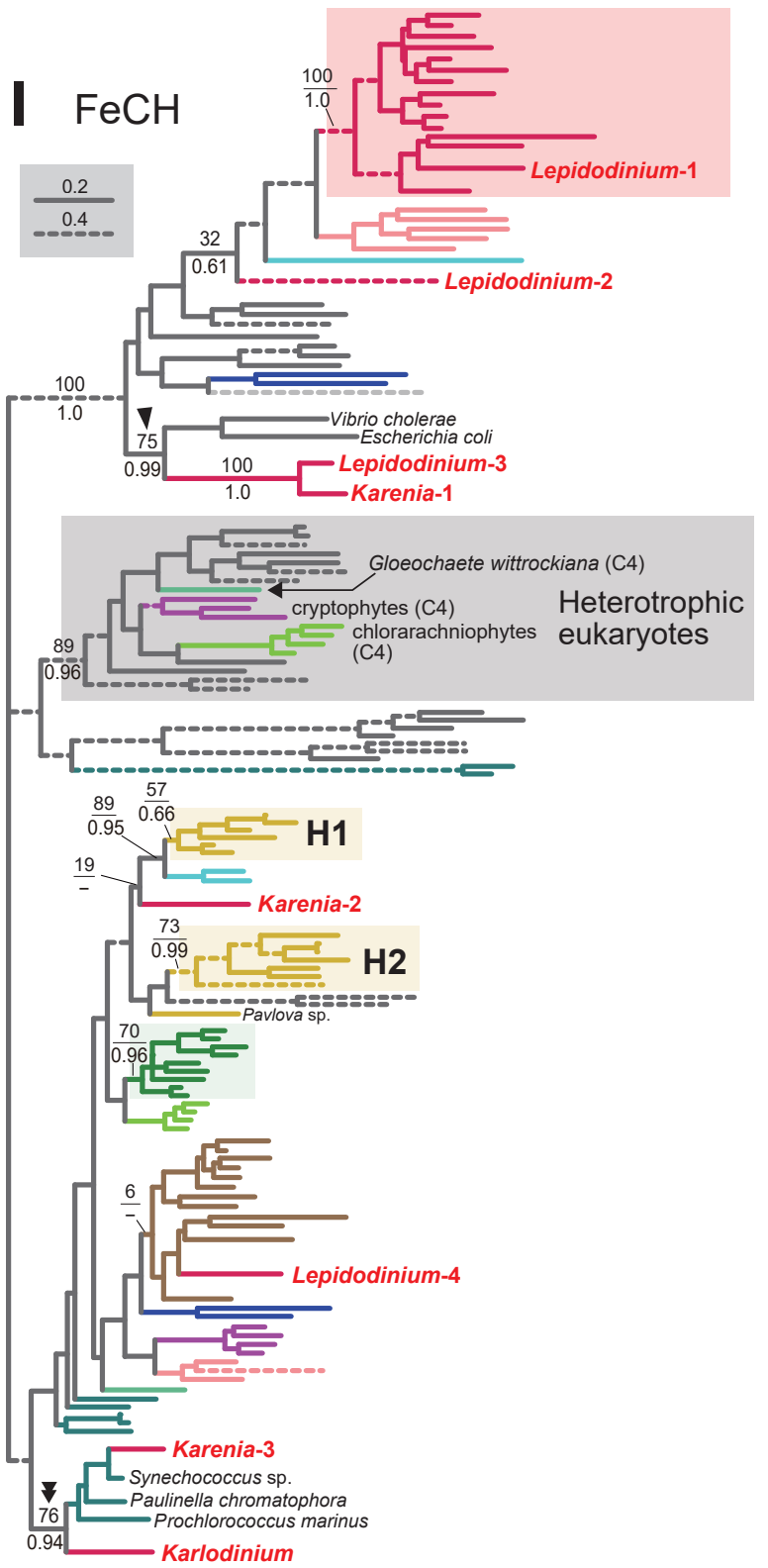


\section{Figure 3 (on next page)}

Maximum-likelihood phylogenies of 7 proteins involved in the $\mathrm{Chl}$ a biosynthetic pathway.

The details of this figure are same as those of Fig. 2. A. ChID, one of the two nucleusencoded subunits of $\mathrm{Mg}$-chelatase $(\mathrm{MgCH})$. B. $\mathrm{ChlH}$, the other nucleus-encoded subunit of $\mathrm{MgCH}$. C. S-adenosylmethionine:Mg-protoporphyrin O-methyltransferase (MgPMT). D. divinyl chlorophyllide a 8-vinyl-reductase using ferredoxin for electron donor (F-DVR). E. divinyl chlorophyllide a 8-vinyl-reductase using NADPH for electron donor (N-DVR). F. lightdependent protochlorophyllide reductase (POR). G. chlorophyll synthase (CS). The identical $M L$ trees with full sequence names and MLBPs $\geq 50 \%$ are provided as the supplementary materials. Note that we present no phylogeny of Chll or MgPME cyclase, as the former is plastid-encoded, and the latter was not identified in dinoflagellates (regardless of plastidtype), diatoms, cryptophytes or haptophytes. Note that the substitution rates of the dashed branches are different from that of the solid lines as indicated in each figure. 


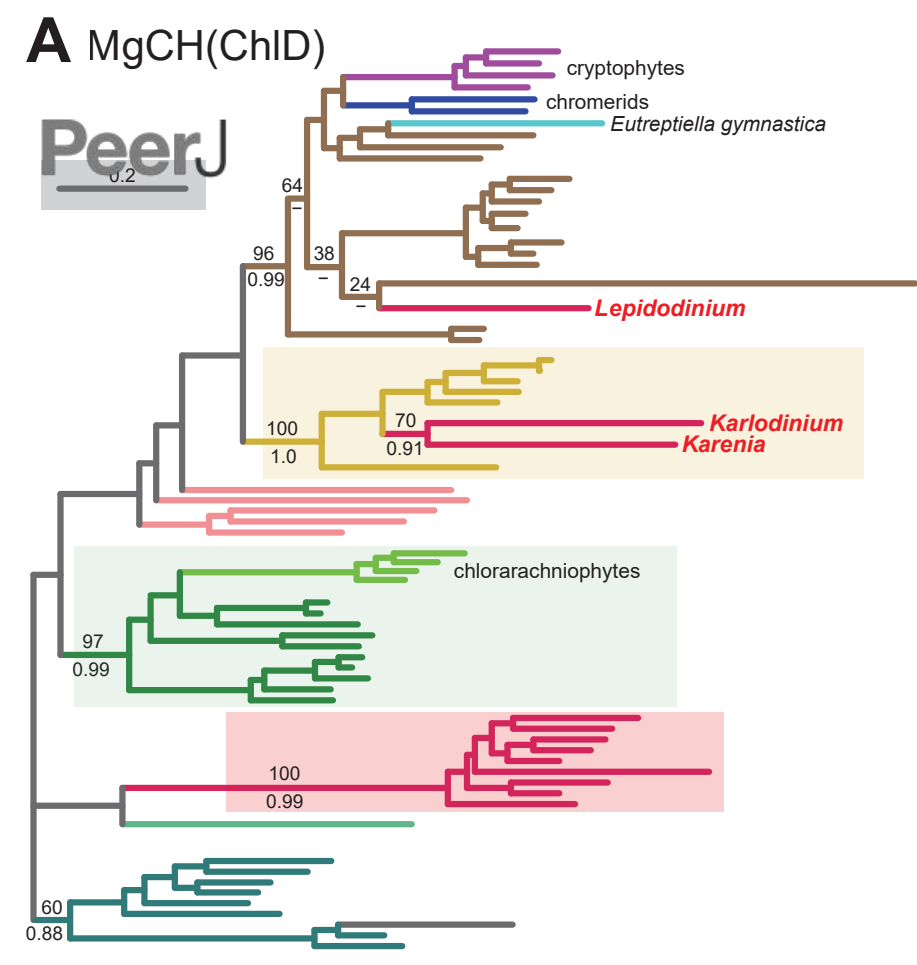

B $\mathrm{MgCH}(\mathrm{ChlH})$
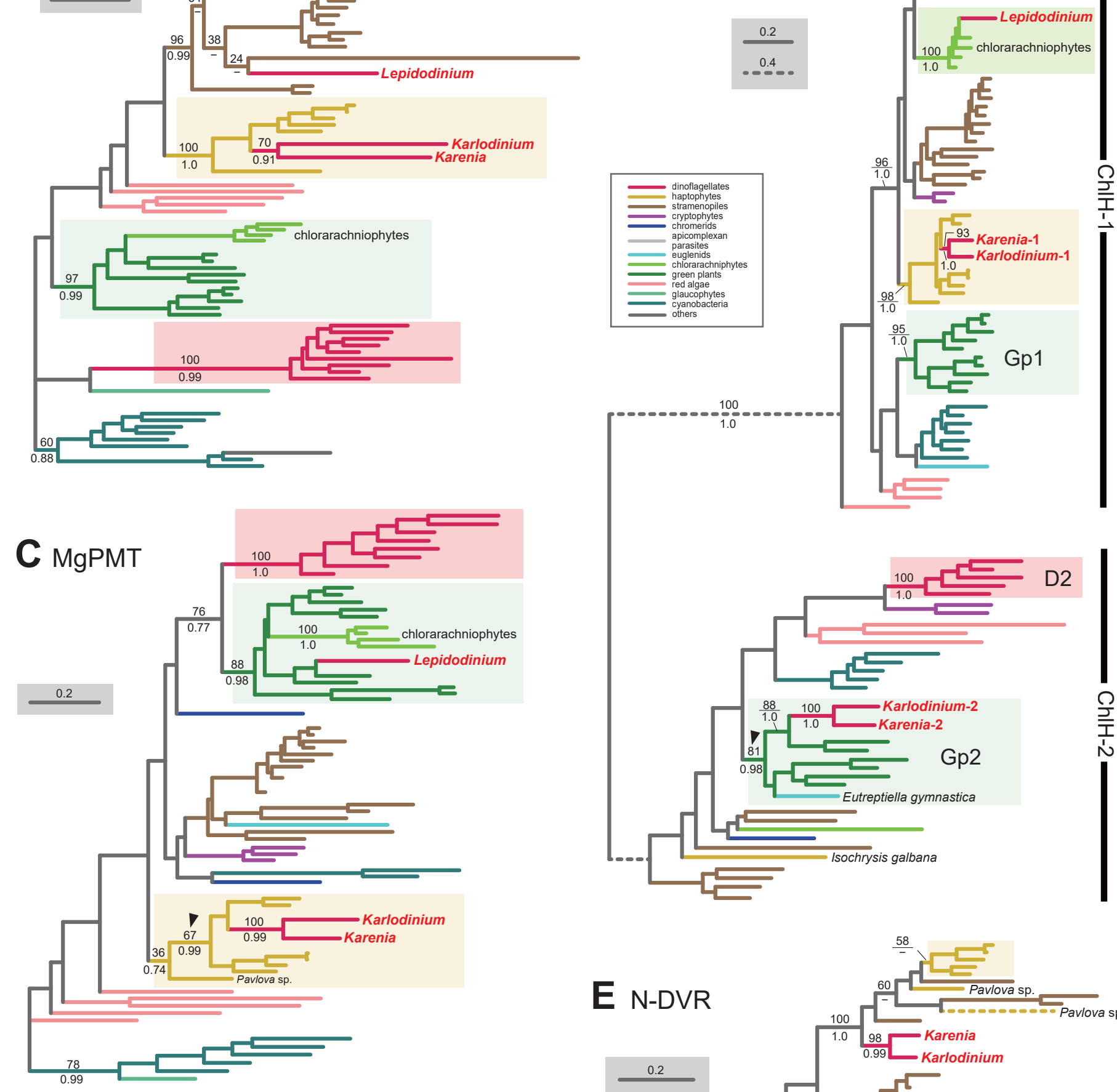

Manuscript to pe revited

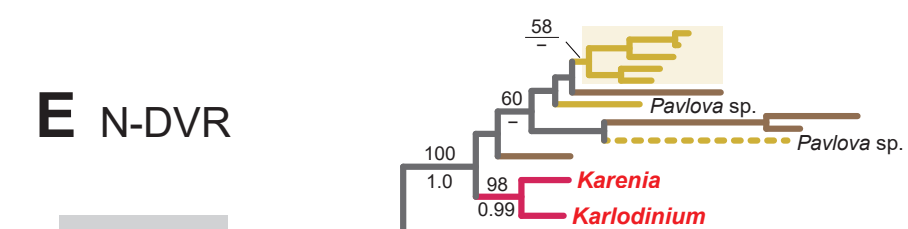

D F-DVR

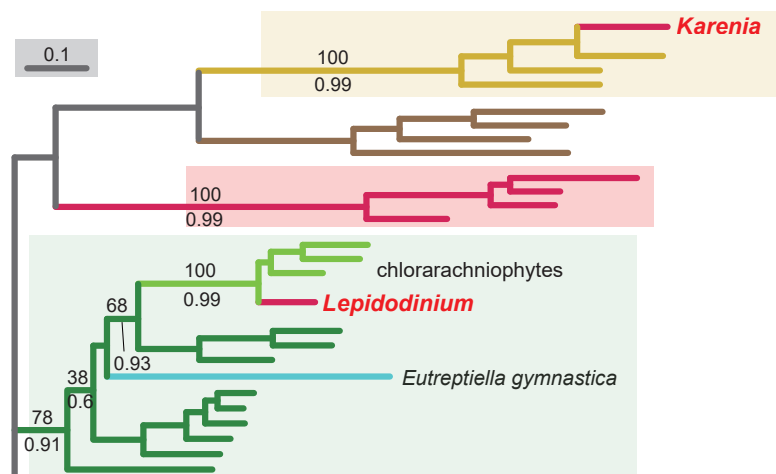

PeerJ reviewtigPF | (2017:11:21975:2:0:NEW 27 Jun 2018)
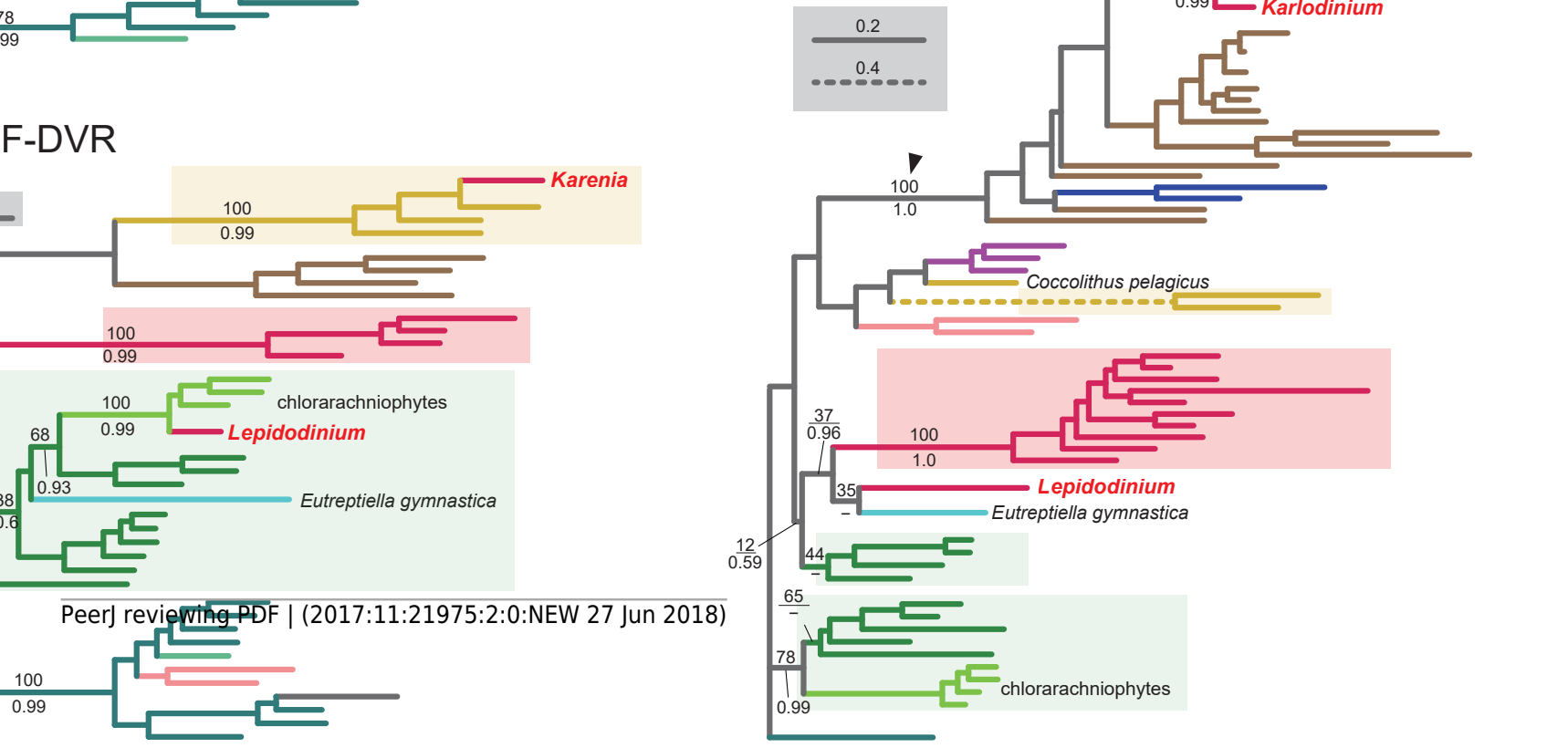
PeerJ

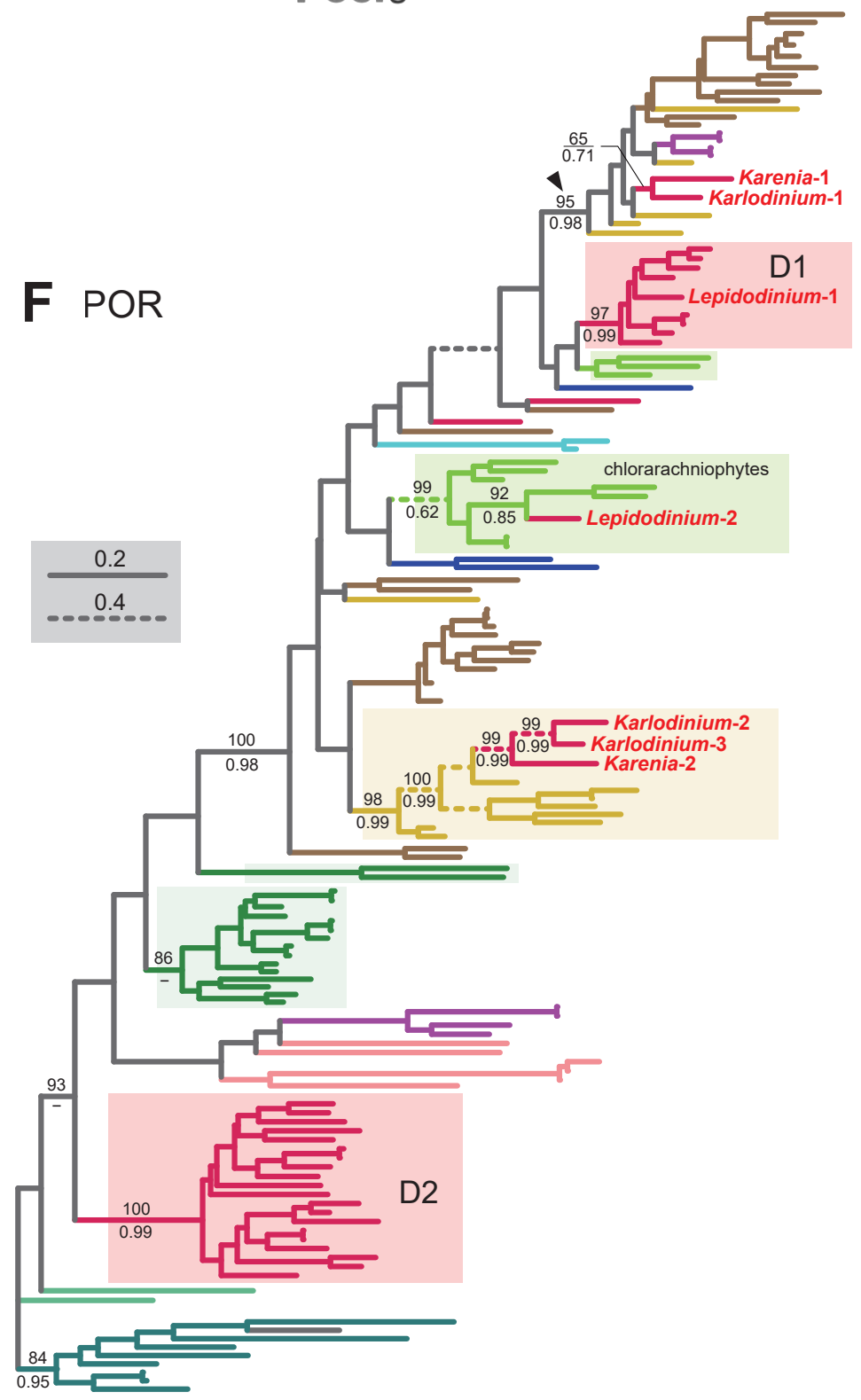

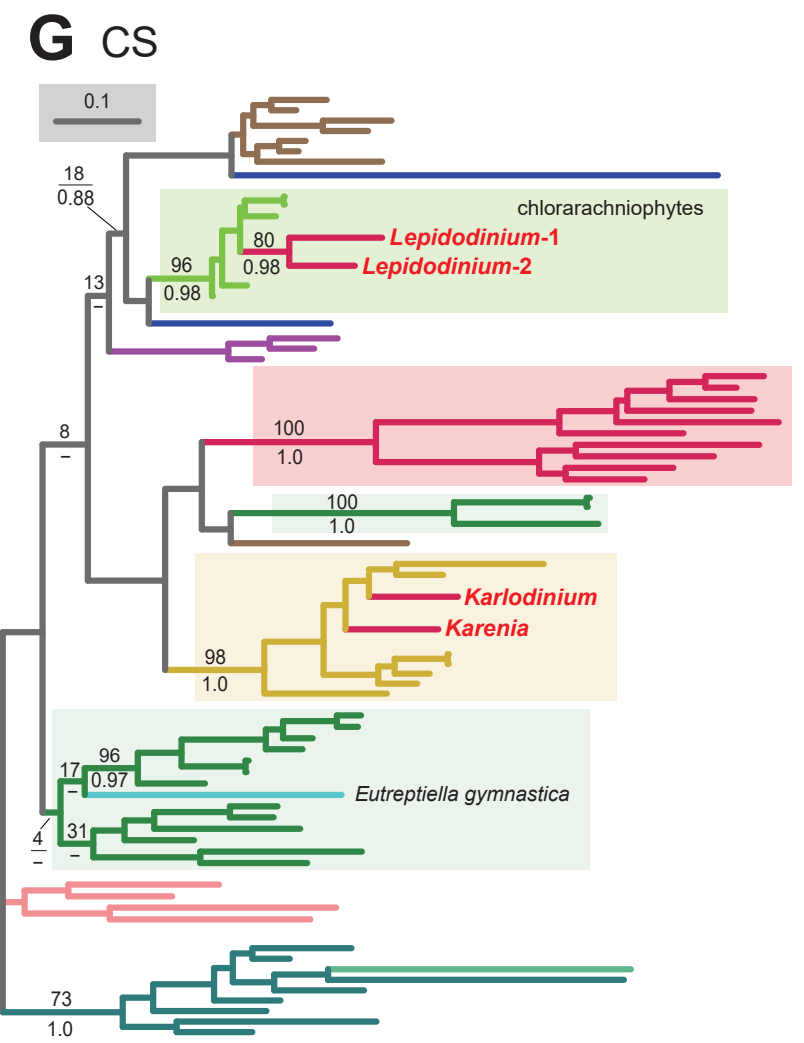

PeerJ reviewing PDF | (2017:11:21975:2:0:NEW 27 Jun 2018) 


\section{Figure 4 (on next page)}

Maximum-likelihood phylogenies of 7 proteins involved in the non-mevalonate pathway for IPP biosynthesis.

The details of this figure are same as those of Fig. 2. A. 1-deoxy-D-xylulose-5-phosphate (DXP) synthase (DXS). B. DXP reductoisomerase (DXR). C. 2-C-methyl-D-erythritol 4phosphate cytidylyltransferase (IspD). D. 4-diphosphocytidyl-2-C-methyl-D-erythritol kinase (IspE). E. 2-C-methyl-D-erythritol 2,4-cyclodiphosphate synthase (IspF). F. 1-hydroxy-2methyl-2-butenyl 4-diphosphate (HMB-PP) synthase (IspG). G. HMB-PP reductase (IspH). The identical ML trees with full sequence names and MLBPs $\geq 50 \%$ are provided as the supplementary materials. Note that the substitution rates of the dashed branches are different from that of the solid lines as indicated in each figure. 




\section{Figure $\mathbf{5}$ (on next page)}

Overview of the origins of proteins involved in three plastid-localized biosynthetic pathways in Karenia brevis, Karlodinium veneficum and Lepidodinium chlorophorum.

The origins of proteins of interest were classified into three types, (i) "Vl-type" which were vertically inherited from the ancestral dinoflagellate beyond haptophyte/green algal endosymbiosis, (ii) "EA-type" which were acquired from the endosymbiont, and (iii) "LA-type" which were acquired from organisms distantly related to the host (dinoflagellates) or endosymbiont (haptophytes or green algae). Squares indicate the numbers and types of proteins of interest in the three dinoflagellates. In case of multiple versions being identified in one species, the numbers of the versions are shown in the corresponding squares. The squares are color-coded as follows: red, VI-type protein; yellow, EA-type in Karenia brevis and Karlodinium veneficum; green, EA-type in Lepidodinium; grey, LA-type. For DVR involved in the Chl a biosynthetic pathway, we distinguish N-DVR and F-DVR by labeling " $N$ " and " $F$," respectively. The open squares in the fourth lows labeled with question marks represent the sequences of which origins remain uncertain. 


\section{Heme biosynthesis}

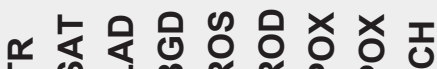

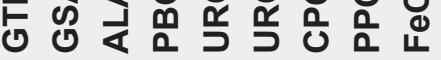

Karenia brevis

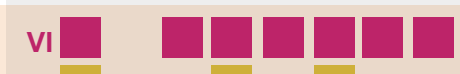

EA LA

?
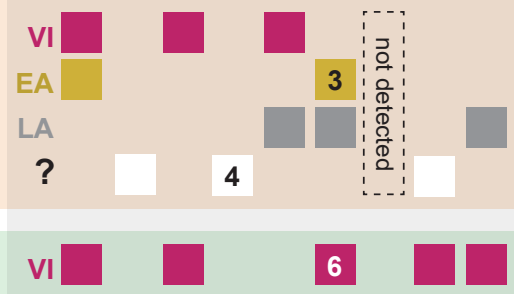

Lepidodinium

chlorophorum

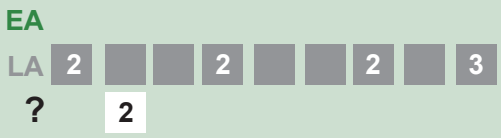

Chl a biosynthesis
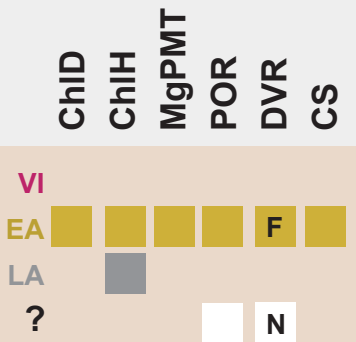

\section{VI}

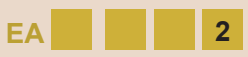

LA

$?$

$\mathbf{N}$

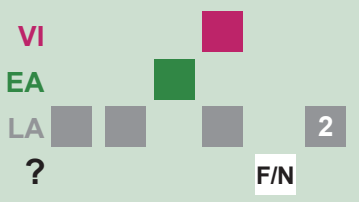

IPP biosynthesis

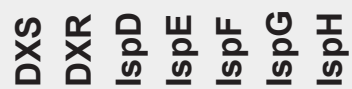
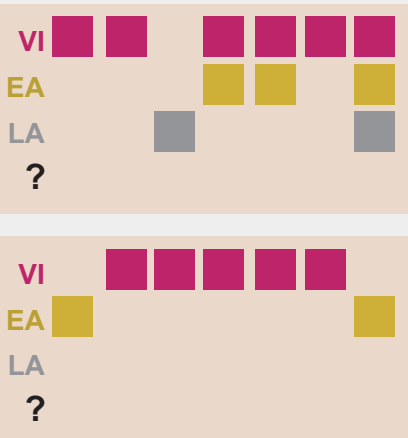

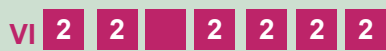
EA

LA 